\title{
SUBCOMPLETIONS OF REPRESENTABLE RELATION ALGEBRAS
}

\author{
ROGER D. MADDUX
}

\begin{abstract}
Many finite symmetric integral non-representable relation algebras, including almost all Monk algebras, can be embedded in the completion of an atomic symmetric integral representable relation algebra whose finitelygenerated subalgebras are finite.
\end{abstract}

\section{Introduction}

Monk 26] proved that if $\mathbf{B}$ is a Boolean algebra with operators, then $\mathbf{B}$ has a unique completion $\mathbf{C}$, where $\mathbf{C}$ is a completion of $\mathbf{B}$ if $\mathbf{C}$ is a complete, $\mathbf{B}$ is a subalgebra of $\mathbf{C}$, and $\mathbf{B}$ is dense in $\mathbf{C}$, which means that below every nonzero element of $\mathbf{C}$ there is a non-zero element of $\mathbf{B}$. Monk proved that if $\mathbf{B}$ is a relation algebra algebra, then its completion is also relation algebra. The problem remained, if $\mathbf{B}$ is representable, must its completion also be representable? In other words, is the variety 1 RRA of representable relation algebras closed under completions? Hodkinson [15] provided the answer that, no, RRA is not closed under completions because there is an atomic $\mathbf{B} \in$ RRA such that the completion of $\mathbf{B}$ is not representable 2

Consider any atomic representable relation algebra $\mathbf{B} \in$ RRA whose completion $\mathbf{C}$ is not representable, as might arise from Hodkinson's proof. Since RRA is a variety and $\mathbf{C}$ is not in RRA, there must be an equation $\epsilon$ that holds in RRA but fails in $\mathbf{C}$. Let $\mathbf{A}$ be the subalgebra of $\mathbf{C}$ that is generated by the finitely many values assigned to the variables occurring in $\epsilon$. Then $\mathbf{A}$ is also not representable because it fails to satisfy $\epsilon$. Thus $\mathbf{A}$ is an example of a finitely-generated relation algebra which is a subalgebra of the non-representable completion of an atomic RRA. The question addressed by this paper is, which relation algebras can occur as A? We rephrase this question as a problem.

Problem 1. Let $K$ be the class of finitely-generated subalgebras of non-representable completions of atomic representable relation algebras. Which relation algebras are in $K$ ? Does $K$ contain any relation algebras that are not weakly representable?

Date: August 3, 2011.

1991 Mathematics Subject Classification. 03G15.

Key words and phrases. Relation algebras, cylindric algebras, Monk algebras, completion, representable, finite-variable logic, algebraic logic.

${ }^{1}$ It is easy to show that RRA is closed under subalgebras and direct products. Closure under homomorphic images was first proved by Tarski [33] using model theory; for a more direct proof see [24, Th. 121].

${ }^{2}$ For subsequent developments, alternate and simpler constructions, extensions, and related work, see, for example, Andréka-Németi-Sayed Ahmed [2, Hirsch-Hodkinson 9, 10, 11, 12, 13, Hodkinson-Venema [16], Khaled-Sayed Ahmed [17, 18, Sayed Ahmed 27, 28, 29, 30, 31, and Sayed Ahmed-Samir 32 . 
Some finite symmetric integral relation algebras have no proper extensions at all and are therefore neither representable nor in $K$; see Frias-Maddux [6] for examples.

As a partial positive answer, it will be shown in this paper that every finite Monk algebra with six or more colors is in $K$. Monk algebras are relation algebraic versions of cylindric algebras used by Monk [25] to prove that classes of finite-dimensional representable cylindric algebras are not finitely axiomatizable.

\section{Monk algebras}

Definition 1. For $4 \leq q \in \omega$, the no 1-cycles algebra $\mathbf{E}_{q}^{23}$ is the finite symmetric integral relation algebra with $q$ atoms $\mathbf{e}_{0}=1$ ', $\mathbf{e}_{1}, \cdots, \mathbf{e}_{q-1}$ such that if $a, b$ are distinct diversity atoms then $a ; b=0$ ' and $a ; a=\bar{a}$.

These relation algebras were constructed in [21] and were called $\mathfrak{E}_{q}(\{2,3\})$ in $[23$, Def. 2.4, Prob. 2.7]. Two particular examples of these algebras, namely $\mathbf{E}_{4}^{23}$ and $\mathbf{E}_{5}^{23}$, are $62_{65}$ and $3009_{3013}$, respectively, in [24]. It is likely that $\mathbf{E}_{q}^{23}$ is representable for all $q \geq 4$. This is known to be true for $q=4$; the earliest reference to the representability on a 13 -element set of $\mathbf{E}_{4}^{23}$ is in a footnote by Lyndon [19]. In fact, $\mathbf{E}_{4}^{23}$ is isomorphic to a subalgebra of the complex algebra of the 13-element cycle group $\mathbb{Z}_{13}$, and $\mathbf{E}_{4}^{23}$ has exactly two other square representations, both on a 16-element set. Furthermore, $\mathbf{E}_{q}^{23}$ was shown to be representable by Comer [4] for $q=5,6$ and more recently (by Comer's method) for $q=7,8$.

$\mathbf{E}_{q}^{23}$ can also be described by cycles and atom structures, which are defined for algebras in NA, the class of non-associative relation algebras. An axiom set for NA is obtained by deleting the associative law from the axioms for relation algebras; see [20, Def. 1.2] and [24, Th. 314]. The atom structure [20, Def. 3.2] of an algebra $\mathbf{A} \in \mathrm{NA}$ is $\left\langle A t(\mathbf{A}), C,{ }^{\smile}, I\right\rangle$ where $A t(\mathbf{A})$ is the set of atoms of $\mathbf{A}, C$ is the set of triples of atoms $\langle x, y, z\rangle$ such that $x ; y \geq z,{ }^{\sim}$ is the restriction of the converse operation of $\mathbf{A}$ to the atoms of $\mathbf{A}$, and $I=\left\{x: 1^{\prime} \geq x \in A t(\mathbf{A})\right\}$. In every NA, $C$ is the union of sets of the form

$$
[x, y, z]=\{\langle x, y, z\rangle,\langle\breve{x}, z, y\rangle,\langle y, \breve{z}, \breve{x}\rangle,\langle\breve{y}, \breve{x}, \breve{z}\rangle,\langle\breve{z}, x, \breve{y}\rangle,\langle z, \breve{y}, x\rangle\},
$$

where $x, y, z \in A t(\mathbf{A})$. Such sets are called cycles. If 1 ' is an atom of $\mathbf{A}$, then the cycle $[x, y, z]$ is said to be an identity cycle if $1^{\prime} \in[x, y, z]$, and a diversity cycle otherwise. If $\mathbf{A}$ is symmetric, i.e., $\breve{x}=x$ for all $x$, then a diversity cycle $[x, y, z]$ is said to be a 1-cycle, 2-cycle, or 3-cycle if the cardinality $|\{x, y, z\}|$ is 1 , 2, or 3 , respectively. For example, the cycles of $\mathbf{E}_{q}^{23}$ are all the 2-cycles and 3-cycles, but none of the 1-cycles.

Definition 2 (Andréka-Maddux-Németi [1]). Let $\mathbf{A}$ and $\mathbf{B}$ be atomic relation algebras. We say that $\mathbf{A}$ is obtained from $\mathbf{B}$ by splitting if $\mathbf{B} \subseteq \mathbf{A}$, every atom $x$ of $\mathbf{A}$ is contained in an atom $\mathrm{c}(x)$ of $\mathbf{B}$, called the cover of $x$, and for all $x, y \in$ At $\mathbf{A}$, if $x, y \leq 0$ ' then

$$
x ; y= \begin{cases}\mathrm{c}(x) ; \mathrm{c}(y) \cdot 0, & \text { if } x \neq \breve{y} \\ \mathrm{c}(x) ; \mathrm{c}(y) & \text { if } x=\breve{y} .\end{cases}
$$

Definition 3 (Andréka-Maddux-Németi [1, Ex. 6]). A Monk algebra is an atomic symmetric integral relation algebra obtained by splitting from some $\mathbf{E}_{q}^{23}, 4 \leq q \in \omega$.

Assume $\mathbf{A}$ is a Monk algebra obtained from $\mathbf{E}_{q}^{23}$ by splitting. Then $\mathbf{A}$ extends $\mathbf{E}_{q}^{23}$ and the $q-1$ diversity atoms of the subalgebra $\mathbf{E}_{q}^{23} \subseteq \mathbf{A}$ are called the colors 
of $\mathbf{A}$. Consider a subalgebra $\mathbf{E} \subseteq \mathbf{E}_{q}^{23} \subseteq \mathbf{A}$. Then the Monk algebra $\mathbf{A}$ extends its subalgebra $\mathbf{E}$ in a way that, for want of a better name, we simply call "special".

Definition 4. If $\mathbf{A}$ and $\mathbf{E}$ are finite symmetric integral relation algebras, then $\mathbf{A}$ is said to be a special extension of $\mathbf{E}$ if $\mathbf{E} \subseteq \mathbf{A}$ and for all diversity atoms $0^{\prime} \geq a, b, c \in A t(\mathbf{E})$,

(i) if not $(a=b=c)$ and $a ; b \geq c$ then $x ; y \geq c$ whenever $a \geq x \in \mathbf{A t}(\mathbf{A})$ and $b \geq y \in \mathbf{A t}(\mathbf{A})$,

(ii) if $a ; a \geq a$ then $x ; y \cdot a \neq 0$ whenever $a \geq x, y \in \mathbf{A t}(\mathbf{A})$.

Every finite symmetric integral relation algebra is a special extension of itself. Every finite symmetric integral relation algebra with no functional atom: $3^{3}$ is also a special extension of its minimum subalgebra, the one whose atoms are 1' and 0'.

Lemma 1. Every Monk algebra obtained from $\mathbf{E}_{q}^{23}$ by splitting is a special extension of every subalgebra of $\mathbf{E}_{q}^{23}$.

Proof. Assume $\mathbf{E}_{q}^{23} \subseteq \mathbf{A}, 4 \leq q, \mathbf{A}$ is a Monk algebra obtained from $\mathbf{E}_{q}^{23}$ by splitting, and $\mathrm{c}(x)$ is the atom of $\mathbf{E}_{q}^{23}$ containing the atom $x$ of $\mathbf{A}$. Consider a subalgebra $\mathbf{E} \subseteq \mathbf{E}_{q}^{23} \subseteq \mathbf{A}$ and diversity atoms $0^{\prime} \geq a, b, c \in A t(\mathbf{E})$.

To show part (i) of Def.4. we assume not $(a=b=c), a ; b \geq c, a \geq x \in \operatorname{At}(\mathbf{A})$, and $b \geq y \in A t(\mathbf{A})$. We want to prove $x ; y \geq c$, so we also assume $c \geq z \in A t(\mathbf{A})$ and must now show $x ; y \geq z$. Note that $x \leq \mathrm{c}(x) \leq a, y \leq \mathrm{c}(y) \leq b$, and $z \leq \mathrm{c}(z) \leq c$. Also, not $(\mathrm{c}(x)=\mathrm{c}(y)=\mathrm{c}(z))$ since otherwise we would have $a=b=c$, contradicting our assumption. Hence $[\mathrm{c}(x), \mathrm{c}(y), \mathrm{c}(z)]$ is not a 1-cycle, and is either a 2-cycle or 3-cycle. Now $\mathbf{E}_{q}^{23}$ contains all 2-cycles and 3-cycles by definition, so $[\mathrm{c}(x), \mathrm{c}(y), \mathrm{c}(z)]$ is a cycle of $\mathbf{E}_{q}^{23}$, hence $\mathrm{c}(x) ; \mathrm{c}(y) \geq \mathrm{c}(z)$. By definition of splitting, $x$ and $y$ have a product equal to the product of their covers in $\mathbf{E}_{q}^{23}$, so we have $x ; y=\mathrm{c}(x) ; \mathrm{c}(y)$. If $\mathrm{c}(x) \neq \mathrm{c}(y)$ then $x ; y=\mathrm{c}(x) ; \mathrm{c}(y)=0^{\prime} \geq c$ by Def.1. If $\mathrm{c}(x)=\mathrm{c}(y)$ then $a=b$, hence $a=b \neq c$ by the assumption not $(a=b=c)$. Therefore $a \cdot c=0$ since $a, c \in A t(\mathbf{E})$. By Def.1 we get $x ; y=\mathrm{c}(x) ; \mathrm{c}(y)=$ $\mathrm{c}(x) ; \mathrm{c}(x)=\overline{\mathrm{c}(x)} \geq \bar{a} \geq c$.

To show part (ii) of Def. 4. we assume $a \geq x, y \in \mathbf{A t}(\mathbf{A})$ and $a ; a \geq a$. We wish to show that $x ; y \cdot a \neq 0$. Note that $a$ cannot be an atom of $\mathbf{E}_{q}^{23}$ because the assumption $a ; a \geq a$ fails for all atoms of $\mathbf{E}_{q}^{23}$ by Def.1. Assume first that $\mathrm{c}(x)=\mathrm{c}(y)=u$. Since $a$ is not an atom of $\mathbf{E}, a$ is the join of two or more atoms of $\mathbf{E}_{q}^{23}$, hence there is some atom $v \in A t\left(\mathbf{E}_{q}^{23}\right)$ such that $u \neq v \leq a$. We have $x ; y=\mathrm{c}(x) ; \mathrm{c}(y)=u ; u$ by the definition of splitting, but $u ; u=\bar{u} \geq v$ by Def.1. so $0 \neq x ; y \cdot v \leq x ; y \cdot a$, as desired. Assume that $\mathrm{c}(x) \neq \mathrm{c}(y)$. Then $\mathrm{c}(x) ; \mathrm{c}(y)=0$ ' by Def.[1, so $x ; y=\mathrm{c}(x) ; \mathrm{c}(y)=0^{\prime} \geq a$, hence $x ; y \cdot a \neq 0$.

Lemma 1 suggests that we consider an arbitrary subalgebra $\mathbf{E}$ of $\mathbf{E}_{q}^{23}$. Every subalgebra contains 1', but 1' is an atom in $\mathbf{E}_{q}^{23}$, so it is an atom in $\mathbf{E}$ as well. Thus $\mathbf{E}$ is integral, but $\mathbf{E}$ is also symmetric since it is the subalgebra of a symmetric algebra. The diversity atoms of $\mathbf{E}$ are disjoint and join up to 0', so they partition the diversity atoms of $\mathbf{E}_{q}^{23}$. In every relation algebra, the relative product $a ; b$ of distinct diversity atoms $a, b$ of $\mathbf{E}$ is included in 0 '. On the other hand, in this case we have $a ; b \geq 0$ ' because there are atoms $x, y$ of $\mathbf{E}_{q}^{23}$ such that $a \geq x, b \geq y$, and $a ; b \geq x ; y=0$ ' by Def.1. Every diversity atom of $a \in \mathbf{E}$ satisfies either $a ; a=1$

\footnotetext{
${ }^{3}$ A functional atom $x$ in a symmetric integral relation algebra satisfies $x ; x=1$ '.
} 
or $a ; a=\bar{a}$, for if $a$ is an atom of $\mathbf{E}_{q}^{23}$ (as well as $\mathbf{E}$ ) then $a ; a=\bar{a}$ by Def.1, while if $a$ is not an atom of $\mathbf{E}_{q}^{23}$, then it is the join of two or more atoms of $\mathbf{E}_{q}^{23}$, say $a \geq \mathbf{e}_{1}+\mathbf{e}_{2}$, so

$$
\begin{aligned}
a ; a & \geq\left(\mathbf{e}_{1}+\mathbf{e}_{2}\right) ;\left(\mathbf{e}_{1}+\mathbf{e}_{2}\right) \\
& =\mathbf{e}_{1} ; \mathbf{e}_{1}+\mathbf{e}_{1} ; \mathbf{e}_{2}+\mathbf{e}_{2} ; \mathbf{e}_{1}+\mathbf{e}_{2} ; \mathbf{e}_{2} \\
& =\overline{\mathbf{e}_{1}}+0^{\prime}+0^{\prime}+\overline{\mathbf{e}_{2}} \\
& =1 .
\end{aligned}
$$

Every subalgebra of $\mathbf{E}_{q}^{23}$ can therefore be characterized by just two parameters: $\alpha$, the number of diversity atoms $a$ satisfying $a ; a=\bar{a}$, and $\beta$, the number of diversity atoms satisfying $a ; a=1$. The number of atoms in the subalgebra is $1+\alpha+\beta$. The only restrictions on these parameters are $\alpha+2 \beta<q$ and $0<\alpha+\beta$.

An atom $a$ of a symmetric integral relation algebra is said to be flexible if $a ; a=1$ and $x ; a=0$ ' for all diversity atoms $x$ distinct from $a$. Having a flexible atom is a sufficient condition for representability; see Comer [5, 5.3] or [22, Th.6]. Since every proper subalgebra of $\mathbf{E}_{q}^{23}$ has at least one atom $a$ satisfying $a ; a=1$ and this atom is flexible by Def.1, every proper subalgebra of $\mathbf{E}_{q}^{23}$ is representable.

\section{An infinite atom structure from two finite algebras}

In this section we use an arbitrary finite symmetric integral relation algebra $\mathbf{A}$ and its subalgebra $\mathbf{E}$ to construct a complete atomic algebra $C_{\mathbf{E}}(\mathbf{A}) \in \mathrm{NA}$ that has subalgebras isomorphic to $\mathbf{A}$ and $\mathbf{E}$.

The complex algebra of the structure $\left\langle A, C,{ }^{\smile}, I\right\rangle$, where $A$ is a set, $C$ is a ternary relation on $A,{ }^{\smile}$ is a unary operation on $A$, and $I \subseteq A$, is the Boolean algebra of all subsets of $A$ supplemented with $I$ as a distinguished element, the unary complex converse operation defined by $\breve{X}=\{\breve{x}: x \in X\}$ for all $X \subseteq A$, and the complex relative multiplication defined by

$$
X ; Y=\{z: \exists x \in X, \exists y \in Y,\langle x, y, z\rangle \in C\}
$$

for all $X, Y \subseteq A$. Every complete atomic NA is isomorphic to the complex algebra of its atom structure [20, Th.3.13(2)]. Therefore, to define a complete atomic NA, as we do in the following definition, it is enough to describe its atom structure.

Definition 5. Assume $\mathbf{E} \subseteq \mathbf{A} \in \mathrm{RA}$ are finite symmetric integral relation algebras. Then $C_{\mathbf{E}}(\mathbf{A})$ is the complete atomic NA with this atom structure: the atoms of $C_{\mathbf{E}}(\mathbf{A})$ are 1 ' and the ordered pair $x^{(i)}$ for every diversity atom $x$ of $\mathbf{A}$ and every index $i \in \omega$,

$$
\operatorname{At}\left(C_{\mathbf{E}}(\mathbf{A})\right):=\left\{1^{\prime}\right\} \cup\left\{x^{(i)}: 0^{\prime} \geq x \in \operatorname{At}(\mathbf{A}), i \in \omega\right\},
$$

the converse of every atom is itself, if $T \subseteq \omega^{3}$ is defined for $i, j, k \in \omega$ by

$$
T(i, j, k) \Longleftrightarrow(i \leq j=k) \vee(j \leq k=i) \vee(k \leq i=j),
$$

and $\mathrm{c}(x)$ is the atom of $\mathbf{E}$ containing the atom $x$ of $\mathbf{A}$, then the cycles of $C_{\mathbf{E}}(\mathbf{A})$ are, for all $0^{\prime} \geq x, y, z \in A t(\mathbf{A})$ and $i, j, k \in \omega$,

$$
\begin{aligned}
& {\left[1^{\prime}, 1^{\prime}, 1^{\prime}\right], \quad\left[1^{\prime}, x^{(i)}, x^{(i)}\right],} \\
& {\left[x^{(i)}, y^{(j)}, z^{(k)}\right] \quad \text { if } x ; y \geq z \wedge(\mathrm{c}(x)=\mathrm{c}(y)=\mathrm{c}(z) \Rightarrow T(i, j, k)) .}
\end{aligned}
$$


For any $a \in A$ and $n \in \omega$, define the element $J(a, n)$ of $C_{\mathbf{E}}(\mathbf{A})$ by

$$
J(a, n)=\sum\left\{x^{(i)}: 0^{\prime} \cdot a \geq x \in A t(\mathbf{A}), n \leq i \in \omega\right\}+\sum\left\{1^{\prime}: 1^{\prime} \leq a\right\},
$$

so if $x$ is an atom of $\mathbf{A}$, then

$$
J(x, n)= \begin{cases}\sum\left\{x^{(i)}: n \leq i \in \omega\right\} & \text { if } x \leq 0 \\ 1 & \text { if } x=1\end{cases}
$$

(i) if $0^{\prime} \geq x \in \operatorname{At}(\mathbf{A}), \mathrm{c}(x)=a \in \operatorname{At}(\mathbf{E}), i, j \in \omega$, and $i \neq j$, then

$$
\begin{aligned}
& x^{(i)} ; x^{(i)}=J\left(0^{\prime} \cdot \bar{a} \cdot x ; x, 0\right)+\sum\left\{z^{(k)}: k \leq i, a \cdot x ; x \geq z \in A t(\mathbf{A})\right\}+1^{\prime}, \\
& x^{(i)} ; x^{(j)}=J\left(0^{\prime} \cdot \bar{a} \cdot x ; x, 0\right)+\sum\left\{z^{(\max (i, j))}: a \cdot x ; x \geq z \in \operatorname{At}(\mathbf{A})\right\},
\end{aligned}
$$

(ii) if $0^{\prime} \geq x, y \in A t(\mathbf{A}), i, j \in \omega$, and $\mathrm{c}(x) \neq \mathrm{c}(y)$ then

$$
x^{(i)} ; y^{(j)}=J(x ; y, 0)=\sum\left\{z^{(k)}: x ; y \geq z \in A t(\mathbf{A}), k \in \omega\right\},
$$

(iii) if $0^{\prime} \geq x, y \in \operatorname{At}(\mathbf{A}), x \neq y, i, j \in \omega, i \neq j$, and $\mathrm{c}(x)=\mathrm{c}(y)=a \in \operatorname{At}(\mathbf{E})$ then

$$
\begin{aligned}
& x^{(i)} ; y^{(j)}=J\left(0^{\prime} \cdot \bar{a} \cdot x ; y, 0\right)+\sum\left\{z^{(\max (i, j))}: a \cdot x ; y \geq z \in A t(\mathbf{A})\right\}, \\
& x^{(i)} ; y^{(i)}=J\left(0^{\prime} \cdot \bar{a} \cdot x ; y, 0\right)+\sum\left\{z^{(k)}: k \leq i, a \cdot x ; y \geq z \in A t(\mathbf{A})\right\} .
\end{aligned}
$$

Start with a finite symmetric integral relation algebra $\mathbf{A}$ in which every atom is splittable (in the sense of [1). Let $\mathbf{A}^{\omega} \supseteq \mathbf{A}$ be the relation algebra obtained by splitting every atom $a \in A t(\mathbf{A})$ into $\omega$ pieces $a^{(0)}, a^{(1)}, \cdots$ so that $a=\sum_{i \in \omega} a^{(i)}$. Splitting produces the maximum set of cycles in the extension $\mathbf{A}^{\omega} \supseteq \mathbf{A}$ that are consistent with containing $\mathbf{A}$ as a subalgebra. Let $\mathbf{E} \subseteq \mathbf{A}$ be a subalgebra of $\mathbf{A}$. From the atom structure of $\mathbf{A}^{\omega}$ we obtain a new atom structure whose complex algebra is, in fact, isomorphic to $C_{\mathbf{E}}(\mathbf{A})$, by deleting all the diversity cycles $\left[a^{(i)}, b^{(j)}, c^{(k)}\right]$ of $\mathbf{A}^{\omega}$ which have the property that all the atoms in the cycle lie below the same atom of $\mathbf{E}$, and $T(i, j, k)$ fails to hold. This leaves only a "thin" remnant of the cycles of $\mathbf{A}^{\omega}$ that we would classify as "1-cycles of $\mathbf{E}$ " (because their atoms all lie below a single atom of $\mathbf{E}$ ). The set of 1-cycles produced by splitting is significantly reduced by imposing the "thinning condition" $T(i, j, k)$. Those cycles of $\mathbf{A}$ that are "covered" by 1-cycles of $\mathbf{E}$ are "thinly reproduced" in $C_{\mathbf{E}}(\mathbf{A})$, while the 2 - and 3 -cycles of $\mathbf{A}$ that are covered by 2- or 3-cycles of $\mathbf{E}$ are "split" into as many cycles as possible. Treating 1-, 2-, and 3-cycles differently in various combinations, either thinning or splitting each type of cycle, gives six more constructions that perhaps should be examined with regard to Problem 1 .

Lemma 2. A is isomorphic, by $a \mapsto J(a, n)$, to a subalgebra $\mathbf{A}^{\prime}$ of $C_{\mathbf{E}}(\mathbf{A})$.

$$
\mathbf{A} \cong \mathbf{A}^{\prime} \subseteq C_{\mathbf{E}}(\mathbf{A}) .
$$

Proof. Define the function $\varphi: \mathbf{A} \rightarrow C_{\mathbf{E}}(\mathbf{A})$ by $\varphi(a)=J(a, 0)$ for all $a \in A$. For a key part of the proof that $\varphi$ embeds $\mathbf{A}$ into $C_{\mathbf{E}}(\mathbf{A})$, assume $0^{\prime} \geq x, y \in \operatorname{At}(\mathbf{A})$. We wish to prove that $\varphi(x) ; \varphi(y)$ and $\varphi(x ; y)$ contain the same diversity atoms of $C_{\mathbf{E}}(\mathbf{A})$. (Proofs for the other parts, involving preservation by $\varphi$ of the Boolean structure and identity element, are fairly easy.)

Consider an arbitrary diversity atom $z^{(k)} \in A t\left(C_{\mathbf{E}}(\mathbf{A})\right)$, where $0^{\prime} \geq z \in A t(\mathbf{A})$, $k \in \omega$. Assume $z^{(k)} \leq \varphi(x) ; \varphi(y)$. Then there are $u, v \in A t\left(C_{\mathbf{E}}(\mathbf{A})\right)$ such that 
$z^{(k)} \leq u ; v, \varphi(x) \geq u \in A t\left(C_{\mathbf{E}}(\mathbf{A})\right)$, and $\varphi(y) \geq v \in A t\left(C_{\mathbf{E}}(\mathbf{A})\right)$. By (8) there are some $i, j \in \omega$ such that $u=x^{(i)}$ and $v=y^{(j)}$. But then $\left[x^{(i)}, y^{(j)}, z^{(k)}\right]$ is a cycle of $C_{\mathbf{E}}(\mathbf{A})$, so $x ; y \geq z$ in $\mathbf{A}$, which implies $z^{(k)} \leq J(x ; y, 0)$, hence $z^{(k)} \leq \varphi(x ; y)$. The argument is reversible.

Every diversity atom in $\mathbf{A}^{\prime}$ is the join of an infinite set of atoms. Therefore $C_{\mathbf{E}}(\mathbf{A})$ cannot be a relation algebra if $\mathbf{A}$ has any diversity atoms that are not splittable. In fact, $C_{\mathbf{E}}(\mathbf{A})$ satisfies all the axioms for relation algebras except possibly the associative law, so $C_{\mathbf{E}}(\mathbf{A}) \in \mathrm{NA}$.

Here is a computational lemma needed several times later.

Lemma 3. Assume $\mathbf{A}$ is a special extension of $\mathbf{E}, a, b$ are distinct diversity atoms of $\mathbf{E}$, and $u, v$ are diversity atoms of $C_{\mathbf{E}}(\mathbf{A})$. If $u \leq J(a, 0)$ and $v \leq J(b, 0)$ then $u ; v=J(a ; b, 0)$. In particular, if $a ; b=0$, then $u ; v=0$ '.

Proof. From $u \leq J(a, 0)$ and $v \leq J(b, 0)$ we have $x^{(i)}=u, y^{(j)}=v, x \leq a=\mathrm{c}(x)$, and $y \leq b=\mathrm{c}(y)$, for some $x, y \in A t(\mathbf{A})$ and $i, j \in \omega$. The covers of $x$ and $y$ are different because $x \leq \mathrm{c}(x)=a \neq b=\mathrm{c}(y) \geq y$. Rule Def.[5] (ii)] applies in this case and says that $x^{(i)} ; y^{(j)}=J(x ; y, 0)$. Note that $x ; y \leq a ; b$. Since $\mathbf{A}$ is a special extension of $\mathbf{E}$, we deduce from Def.4(i) that every atom of $\mathbf{E}$ below $a ; b$ is also below $x ; y$, hence $x ; y=a ; b$. We conclude that $u ; v=x^{(i)} ; y^{(j)}=J(x ; y, 0)=J(a ; b, 0)$. If $a ; b=0^{\prime}$, then $u ; v=J\left(0^{\prime}, 0\right)$, but $J\left(0^{\prime}, 0\right)$ is the diversity element $0^{\prime}$ of $C_{\mathbf{E}}(\mathbf{A})$, so $u ; v=0$.

\section{Embedding Monk algebras}

Two elements of an atomic relation algebra are said to be almost the same if their symmetric difference is the join of finitely many atoms. We show in Theorem 1 below that if $\mathbf{A}$ is a special extension of $\mathbf{E}$ and $\mathbf{B}$ is the subalgebra of $C_{\mathbf{E}}(\mathbf{A})$ generated by the atoms of $C_{\mathbf{E}}(\mathbf{A})$, then the finitely generated subalgebras of $\mathbf{B}$ are finite and every element of $\mathbf{B}$ almost the same as an element of the subalgebra $\mathbf{E}^{\prime}$ of $\mathbf{B}$ isomorphic to $\mathbf{E}$ by $a \mapsto J(a, 0)$.

For an example, suppose $\mathbf{A}$ is a Monk algebra obtained from $\mathbf{E}_{q}^{23}$ by splitting, $4 \leq q \in \omega$, and $\mathbf{E}$ is a subalgebra of $\mathbf{E}_{q}^{23}$. By Lemma $\mathbf{A}$ is a special extension of $\mathbf{E}$, so Theorem 1 applies to $\mathbf{A}$ and $\mathbf{E}$. Next, we show in Theorem [2)(i)(ii) that if, in addition, $\mathbf{E}$ has a "flexible trio" (Def .8 below) then $\mathbf{B}$ is representable because every finitely generated subalgebra of $\mathbf{B}$ is included in a finite subalgebra of $\mathbf{B}$ that has the "1-point extension property" (Def .6 below). In the example, if $7 \leq q$ (A has at least six colors) then $\mathbf{E}_{q}^{23}$ has a subalgebra $\mathbf{E}$ with a flexible trio, so Theorem [2(i)|(ii) applies, and we conclude that $\mathbf{B} \in$ RRA. Finally, we show in Theorem 2(iii) that if $\mathbf{A}$ has no 1-cycles then $\mathbf{B}$ is not completely representable and $C_{\mathbf{E}}(\mathbf{A})$, the completion of $\mathbf{B}$, is not representable. Theorem [2(iii) applies to A because Monk algebras have no 1-cycles. Cor.1 accordingly says that every finite Monk algebra with six or more colors is a subalgebra of the non-representable completion of an atomic representable relation algebra whose finitely-generated subalgebras are finite.

The conclusion that $C_{\mathbf{E}}(\mathbf{A}) \notin \mathrm{RRA}$ can be obtained without Theorem [2](iii) in case the Monk algebra $\mathbf{A}$ is non-representable, which happens if the number of atoms is large compared to the number of colors. In this case the completion of $\mathbf{B}$ is non-representable simply because it has a non-representable subalgebra (isomorphic to the non-representable Monk algebra A). 
In Theorem 1 we obtain conclusions just from knowing the extension $\mathbf{E} \subseteq \mathbf{A}$ is special. Then in Theorem 2 we also consider what happens when, in addition, $\mathbf{E}$ has a flexible trio and $\mathbf{A}$ has no 1-cycles.

Theorem 1. Assume $\mathbf{A}$ and $\mathbf{E}$ are finite symmetric integral relation algebras, $\mathbf{A}$ is a special extension of $\mathbf{E} \subseteq \mathbf{A}$, and $\mathbf{B} \subseteq C_{\mathbf{E}}(\mathbf{A})$ is the subalgebra of $C_{\mathbf{E}}(\mathbf{A})$ generated by $A t\left(C_{\mathbf{E}}(\mathbf{A})\right)$.

(i) $\mathbf{B}$ is countable, atomic, symmetric, integral, and generated by its atoms.

(ii) $C_{\mathbf{E}}(\mathbf{A})$ and $\mathbf{B}$ have the same atom structure.

(iii) $C_{\mathbf{E}}(\mathbf{A})$ is isomorphic to the complex algebra of the atom structure of $\mathbf{B}$.

(iv) $C_{\mathbf{E}}(\mathbf{A})$ is the completion of $\mathbf{B}$.

(v) There are subalgebras $\mathbf{E}^{\prime} \subseteq \mathbf{A}^{\prime} \subseteq C_{\mathbf{E}}(\mathbf{A})$ with $\mathbf{E}^{\prime} \cong \mathbf{E}$ and $\mathbf{A}^{\prime} \cong \mathbf{A}$.

(vi) Every finitely generated subalgebra of $\mathbf{B}$ is finite.

(vii) Every element of $\mathbf{B}$ is almost the same as an element of $\mathbf{E}^{\prime}$.

Proof. Parts (i) (iv) require only the assumption that $C_{\mathbf{E}}(\mathbf{A})$ is complete and atomic and $\mathbf{B}$ is the subalgebra of $C_{\mathbf{E}}(\mathbf{A})$ generated by the atoms of $C_{\mathbf{E}}(\mathbf{A})$. Everything in parts (i) (iv) is either obvious or very easy to prove; see [20, Th. 3.13] for part (iii), Part (v) was proved in Lemma 2. The assumption that $\mathbf{A}$ is a special extension of $\mathbf{E}$ is needed only for Lemma 4 below, which is used to prove parts (vi) and (vii),

Lemma 4. For every $n \in \omega, B_{n}$ is the set of atoms of a subalgebra of $C_{\mathbf{E}}(\mathbf{A})$, where

(9) $B_{n}=\left\{1^{\prime}\right\} \cup\left\{x^{(i)}: 0^{\prime} \geq x \in \operatorname{At}(\mathbf{A}), n>i \in \omega\right\} \cup\left\{J(a, n): 0^{\prime} \geq a \in \operatorname{At}(\mathbf{E})\right\}$.

Proof. The elements of $B_{n}$ are disjoint and their join is 1 , so the set of joins of subsets of $B_{n}$ is closed under the Boolean operations of $C_{\mathbf{E}}(\mathbf{A})$ and, under those operations, forms a Boolean algebra whose set of atoms is $B_{n}$. The converse of everything in $B_{n}$ is again in $B_{n}$ because conversion is the identity function on $C_{\mathbf{E}}(\mathbf{A})$. What remains is to show the relative product $u ; v$ of any two elements $u, v \in B_{n}$ is the join of a subset of $B_{n}$. For this it is enough to show that every element $w \in B_{n}$ is contained in or disjoint from $u ; v$. This is clearly true whenever $u=1^{\prime}$ or $v=1^{\prime}$ or $w$ is itself an atom of $C_{\mathbf{E}}(\mathbf{A})$, so we may assume $w=J(a, n)$, for some $a \in A t(\mathbf{E})$, and $u+v \leq 0$ '. We will show that if $u ; v$ has nonempty intersection with $J(a, n)$ then $u ; v$ contains $J(a, n)$.

Suppose $u ; v \cdot J(a, n) \neq 0$, where $0^{\prime} \geq u, v \in B_{n}, 0^{\prime} \geq a \in A t(\mathbf{E})$. Then there are $x, y, z \in A t(\mathbf{A})$ and $i, j, k \in \omega$ such that $x^{(i)} \leq u, y^{(j)} \leq v, z^{(k)} \leq J(a, n)$, and $x^{(i)} ; y^{(j)} \geq z^{(k)}$. For both cases below, note that $\mathrm{c}(z)=a$ and $n \leq k$ by Def. (7), and $x ; y \geq z$ by (6).

Case 1. not $(\mathrm{c}(x)=\mathrm{c}(y)=\mathrm{c}(z))$. From $x ; y \geq z$ we get $x ; y \cdot \mathrm{c}(z) \neq 0$ since $0 \neq z \leq \mathrm{c}(z)$, hence $x ; y \geq \mathrm{c}(z)=a$ by Def. [4) The implication in (6) has a false hypothesis and therefore holds trivially in this case for every atom of $\mathbf{A}$ below $a$. It follows by (6) and Def. (7) that $x^{(i)} ; y^{(j)} \geq J(a, 0) \geq J(a, n)$.

Case 2. $\mathrm{c}(x)=\mathrm{c}(y)=\mathrm{c}(z)=a$. In this case, by $x^{(i)} ; y^{(j)} \geq z^{(k)}$ and (6) we have $T(i, j, k)$. From $x^{(i)} \leq u \in B_{n}$ and the relevant definitions, it follows that if $u$ is an atom of $C_{\mathbf{E}}(\mathbf{A})$ then $u=x^{(i)}$ and $i<n$, while if $u$ is not an atom of $C_{\mathbf{E}}(\mathbf{A})$, then $x^{(i)} \leq u=J(a, n)$ since $a=\mathrm{c}(x)$, and $i \geq n$. Consequently, if both $u$ and $v$ were atoms of $C_{\mathbf{E}}(\mathbf{A})$, we would have $i, j<n \leq k$, contrary to $T(i, j, k)$. Hence either $u=J(a, n)$ or $v=J(a, n)$. Since $C_{\mathbf{E}}(\mathbf{A})$ is symmetric, these are 
really the same case. We assume $u=x^{(i)}$ and $v=J(a, n)$, and will prove that $x^{(i)} ; J(a, n) \geq J(a, n)$.

Toward this end, assume $w_{j} \leq J(a, n)$ where $n \leq j \in \omega$ and $w \leq a=\mathrm{c}(w)$. Now $x^{(i)}$ is in $B_{n}$, so $i<n \leq j$, hence $T(i, j, j)$. From $x ; y \geq z$ and $\mathrm{c}(x)=\mathrm{c}(y)=\mathrm{c}(z)=a$ we get $a ; a \cdot a \neq 0$, but $x \leq a$ and $w \leq a$, so $x ; w \cdot a \neq 0$ by Def. L(ii). We may therefore choose an atom $t \in \mathbf{A}$ such that $t \leq x ; w \cdot a$. Then $\mathrm{c}(t)=a$ and $T(i, j, j)$, so $\left[x^{(i)}, t^{(j)}, w^{(j)}\right]$ is a cycle of $C_{\mathbf{E}}(\mathbf{A})$ by $\left([6)\right.$, and $t^{(j)} \leq J(a, n)$ since $t \leq a$ and $n \leq j$, so $w_{j} \leq x^{(i)} ; t^{(j)} \leq x^{(i)} ; J(a, n)$. Since this holds for all atoms $w_{j}$ below $J(a, n)$, we have proved $J(a, n) \leq x^{(i)} ; J(a, n)$.

We have shown that every product of two elements of $B_{n}$ is the join of a subset of $B_{n}$. It follows that $u ; v=\sum\left\{w: u ; v \geq w \in B_{n}\right\}$ for all $u, v \in B_{n}$. Hence,for all $U, V \subseteq B_{n}$, we have

$$
\begin{aligned}
\sum U ; \sum V & =\sum\{u ; v: u \in U, v \in V\} \\
& =\sum\left\{\sum\left\{w: u ; v \geq w \in B_{n}\right\}: u \in U, v \in V\right\} \\
& =\sum\left\{w: u ; v \geq w \in B_{n}, u \in U, v \in V\right\} \\
& \in\left\{\sum X: X \subseteq B_{n}\right\} .
\end{aligned}
$$

Therefore $\left\{\sum X: X \subseteq B_{n}\right\}$ is closed under relative multiplication and is a subalgebra of $C_{\mathbf{E}}(\mathbf{A})$.

We return to the proof of Theorem 1. Suppose $\mathbf{F}$ is a finitely generated subalgebra of $\mathbf{B}$. Since $\mathbf{B}$ is itself generated by $A t\left(C_{\mathbf{E}}(\mathbf{A})\right)$, there is a finite set of atoms $X \subseteq A t\left(C_{\mathbf{E}}(\mathbf{A})\right)$ such that $\mathbf{F}$ is contained in the subalgebra of $C_{\mathbf{E}}(\mathbf{A})$ generated by $X$. Since $X$ is finite and $A t\left(C_{\mathbf{E}}(\mathbf{A})\right) \subseteq \bigcup_{n \in \omega} B_{n}$, we may choose a sufficiently large $n \in \omega$ so that $X \subseteq B_{n}$. Then $\mathbf{F}$ is contained in the subalgebra $\mathbf{B}_{n}$ of $C_{\mathbf{E}}(\mathbf{A})$ generated by $B_{n}$. The subalgebra $\mathbf{B}_{n}$ is finite by the lemma, since its set of atoms is the finite set $B_{n}$, so $\mathbf{F}$ is also finite. Hence (vi) holds. This argument also shows that every element of $\mathbf{B}$ is, for some $n \in \omega$, included in a subalgebra whose set of atoms is $B_{n}$, and hence is a join of elements of $B_{n}$. But every join of elements of $B_{n}$ is almost the same as one of the atoms of $\mathbf{E}^{\prime}$. Hence (vii) holds.

As it happens, every finitely-generated subalgebra of $C_{\mathbf{E}}(\mathbf{A})$ (not just $\mathbf{B}$ ) is also finite, even if the extension $\mathbf{E} \subseteq \mathbf{A}$ is not special. To prove this, one argues that for every finite subset $F$ of $C_{\mathbf{E}}(\overline{\mathbf{A}})$ there is some $n \in \omega$ and some finite partition $\mathcal{P}$ of $\{i: n \leq i \in \omega\}$ such that

$$
\left\{1^{\prime}\right\} \cup\left\{x^{(i)}: x \in A t(\mathbf{A}), n>i \in \omega\right\} \cup\left\{\sum\left\{x^{(i)}: i \in P\right\}: x \in A t(\mathbf{A}), P \in \mathcal{P}\right\}
$$

is the set of atoms of a subalgebra of $C_{\mathbf{E}}(\mathbf{A})$ that contains $F$. The remaining details of this proof are omitted since this fact is not needed and it is also not in itself enough to prove Lemma 4. On the other hand, there is a special case which is easy to prove and needed later.

Lemma 5. Assume $\mathbf{A} \supseteq \mathbf{E}$ are finite symmetric integral relation algebras. For every $n \in \omega$,

$$
\left\{1^{\prime}\right\} \cup\left\{x^{(i)}: 0^{\prime} \geq x \in A t(\mathbf{A}), n>i \in \omega\right\} \cup\left\{J(a, n): 0^{\prime} \geq a \in A t(\mathbf{A})\right\} .
$$

is the set of atoms of a subalgebra of $C_{\mathbf{E}}(\mathbf{A})$. 
Proof. The proof is similar to, but simpler, than the proof of Lemma 4 The closure of the set of joins of subsets of (10) under relative multiplication is an immediate consequence of Def. 馬(i)|(ii)|(iii).

For the next theorem we need some definitions. A relation algebra has the 1point extension property if, loosing speakly, every "finite partial representation" $\mu$ can be extended by one point wherever this is needed. We make this precise as follows.

Definition 6. For any $k \in \omega$ and any atomic relation algebra $\mathbf{A}, B_{k}(\mathbf{A})$ is the set of functions $\mu: k \times k \rightarrow A t(\mathbf{A})$ that satisfy the following conditions.

(B) $\mu_{i, i} \leq 1$ ' for all $i<k$,

(B $\breve{\mu}_{i, j}=\mu_{j, i}$ for all $i, j<k$,

(B) $\mu_{i, l} ; \mu_{l, j} \geq \mu_{i, j}$ for all $i, j, l<k$.

The elements of $B_{k}(\mathbf{A})$ are called basic matrices. A matrix $\mu$ satisfies the identity condition if $\mu_{l, m}=1$ ' iff $l=m$ for all $l, m<k$. We say that $\mathbf{A}$ has the 1-point extension property if, assuming $\mu \in B_{k}(\mathbf{A})$, $\mu$ satisfies the identity condition, $x, y$ are diversity atoms of $\mathbf{A}, i, j<k, i \neq j$, and $\mu_{i, j} \leq x ; y$, there are basic matrix $\mu^{\prime} \in B_{k+1}(\mathbf{A})$ satisfying the identity condition such that $\mu_{l, m}=\mu_{l, m}^{\prime}$ for all $l, m<k, \mu_{i, k}^{\prime}=x$, and $\mu_{k, j}^{\prime}=y$.

Definition 7. A relation algebra $\mathbf{A}$ is completely representable if it has a complete representation, where a representation $\rho$, mapping $\mathbf{A}$ into some algebra of binary relations, is complete if it preserves all joins, i.e., if $X$ is a subset of $\mathbf{A}$ whose join $\sum X$ exists in $\mathbf{A}$, then $\rho\left(\sum X\right)=\bigcup_{x \in X} \rho(x)$.

Definition 8. Three diversity atoms a,b,c of a symmetric integral relation algebra A are said to be a flexible trio if

$$
\begin{aligned}
& a ; a=b ; b=c ; c=1, \\
& a ; b=a ; c=b ; c=0,
\end{aligned}
$$

and, for every atom $x \notin\left\{1^{\prime}, a, b, c\right\}$,

$$
x ; a=x ; b=0, \vee x ; a=x ; c=0 \text { ' } \vee x ; b=x ; c=0 \text { '. }
$$

Theorem 4 (relegated to an Appendix) shows that having a flexible trio is sufficient for representability.

If each of $a, b$, and $c$ is a flexible atom then $a, b, c$ is a flexible trio. Hence any three diversity atoms of $\mathbf{E}_{q}^{23}$ form a flexible trio. It can happen that $a, b, c$ is a flexible trio but none of $a, b, c$ is flexible, as in, for example, the symmetric integral relation algebra with seven atoms $1^{\prime}, a, b, c, d, e, f$ and all diversity cycles except $[a, d, d],[b, e, e]$, and $[c, f, f]$. This algebra has no flexible atoms.

The following theorem applies to all Monk algebras with at least six colors, but many other algebras also satisfy its hypotheses. For an example, let $\mathbf{A}$ be the symmetric integral relation algebra whose atoms are 1', $a_{1}, a_{2}, a_{3}, b_{1}, b_{2}, b_{3}, c_{1}$, $c_{2}$, and $c_{3}$, and whose diversity cycles consist of none of the 1-cycles, all of the 2-cycles, and all the 3 -cycles except $\left[a_{1}, a_{2}, a_{3}\right],\left[b_{1}, b_{2}, b_{3}\right]$, and $\left[c_{1}, c_{2}, c_{3}\right]$. Let $\mathbf{E}$ be the subalgebra of $\mathbf{A}$ whose atoms are 1', $a=a_{1}+a_{2}+a_{3}, b=b_{1}+b_{2}+b_{3}$, and $c=c_{1}+c_{2}+c_{3}$. Then $\mathbf{A}$ is a special extension of $\mathbf{E}, a, b, c$ is a flexible trio of $\mathbf{E}$, and $\mathbf{A}$ has no 1-cycles, so Th. 2 can applied to conclude that $\mathbf{A}$ is in the class $K$ of Problem 1 but A is not a Monk algebra. More than 3000 additional examples can 
be obtained by deleting any or all of the following 2-cycles: $\left[a_{1}, a_{2}, a_{2}\right],\left[a_{2}, a_{3}, a_{3}\right]$, $\left[a_{1}, a_{1}, a_{3}\right],\left[b_{1}, b_{2}, b_{2}\right],\left[b_{2}, b_{3}, b_{3}\right],\left[b_{1}, b_{1}, b_{3}\right],\left[c_{1}, c_{2}, c_{2}\right],\left[c_{2}, c_{3}, c_{3}\right],\left[c_{1}, c_{1}, c_{3}\right]$, and restoring any or all of the deleted 3-cycles, but at least one of the 2- or 3-cycles must be deleted to insure $\mathbf{A}$ is not a Monk algebra. This scheme retains enough 2 -cycles that the extension is always special.

Theorem 2. Assume $\mathbf{E} \subseteq \mathbf{A}$ are finite symmetric integral relation algebras, $\mathbf{A}$ is a special extension of $\mathbf{E}$, and $\mathbf{E}$ has a flexible trio. If $\mathbf{B}$ is the subalgebra of $C_{\mathbf{E}}(\mathbf{A})$ generated by $A t\left(C_{\mathbf{E}}(\mathbf{A})\right)$, then

(i) every finitely generated subalgebra of $\mathbf{B}$ is contained in a subalgebra of $\mathbf{B}$ that has the 1-point extension property,

(ii) $\mathbf{B}$ is representable,

(iii) if $\mathbf{A}$ has no 1-cycles, i.e., $u ; u \cdot u=0$ whenever $1^{\prime} \neq u \in A t(\mathbf{A})$, then $\mathbf{B}$ is not completely representable and the completion of $\mathbf{B}$ is not representable.

Proof. Suppose $\mathbf{F}$ is a finitely generated subalgebra of B. By the argument at the end of the proof of Th.1 there is some $n \in \omega$ such that $\mathbf{F}$ is contained in the subalgebra $\mathbf{B}_{n} \subseteq \mathbf{B}$ with atoms $A t\left(\mathbf{B}_{n}\right)=B_{n}$. Let $a, b, c$ be a flexible trio of $\mathbf{E}$. We will show that $J(a, n), J(b, n), J(c, n)$ is a flexible trio of $\mathbf{B}_{n}$. Consider the product of the first two elements of the trio. Note that $J(a, n) ; J(b, n) \leq 0$ ' since $J(a, n)$ and $J(b, n)$ are disjoint atoms of $\mathbf{B}_{n}$. We have

$$
\begin{aligned}
& J(a, n) ; J(b, n) \\
& =\sum\left\{u ; v: J(a, n) \geq u \in A t\left(C_{\mathbf{E}}(\mathbf{A})\right), J(b, n) \geq v \in A t\left(C_{\mathbf{E}}(\mathbf{A})\right)\right\}
\end{aligned}
$$

but every disjunct $u ; v$ in this last join is 0 ' by Lemma 3 and the assumption $a ; b=0^{\prime}$, so $J(a, n) ; J(b, n)=0^{\prime}$. Similarly, $J(a, n) ; J(c, n)=0^{\prime}=J(b, n) ; J(c, n)$. Thus (12) holds.

For (13), consider a diversity atom of $\mathbf{B}_{n}$ that is not one of $J(a, n), J(b, n), J(c, n)$. It is either an atom of $C_{\mathbf{E}}(\mathbf{A})$ or at atom of $\mathbf{B}_{n}$ with the form $J(d, n)$, where $d$ is a diversity atom of $\mathbf{E}$ distinct from $a, b, c$.

We first consider $J(d, n)$. Now $d$ multiplies to 0 ' with two of $a, b, c$ by (13), say $a ; d=b ; d=0$ '. Choose atoms $x, y$ of $\mathbf{A}$ with $x \leq a$ and $y \leq d$. Then $J(a, n) ; J(d, n) \leq 0$ ' since $J(a, n)$ and $J(d, n)$ are disjoint, and $J(a, n) ; J(d, n) \geq$ $x^{(n)} ; y^{(n)}$, but $x^{(n)} ; y^{(n)}=0$ ' by Lemma 3 because $a ; d=0$ ', so $J(a, n) ; J(d, n)=0$ '. Similarly $J(b, n) ; J(d, n) \geq 0$ ', so the atom $J(d, n)$ multiplies to 0 ' with two of $J(a, n), J(b, n), J(c, n)$, as desired.

Next consider at atom $u$ of $C_{\mathbf{E}}(\mathbf{A})$. It has the form $u=x^{(i)}$ for some diversity atom $x$ of $\mathbf{A}$ and some $i<n$. We claim that the product of $\mathrm{c}(x)$ with (at least) two elements in the trio $a, b, c$ is $0^{\prime}$, say $a ; c(x)=b ; c(x)=0$ '. This follows from (13) if $\mathrm{c}(x)$ is a diversity atom distinct from $a, b, c$, but if $\mathrm{c}(x)$ is one of $a, b, c$, then it follows from (12). Choose an atom $a \geq y \in A t(\mathbf{A})$. Then $x^{(i)} ; J(a, n) \geq x^{(i)} ; y^{(n)}=0$ ' by $a ; d=0$ ' and Lemma 3. Similarly $x^{(i)} ; J(b, n)=0$ ', so the atom $u=x^{(i)}$ multiplies to 0 ' with two of $J(a, n), J(b, n), J(c, n)$, as desired. This finishes the proof of (13) for $J(a, n), J(b, n), J(c, n)$.

For (11), we will prove $J(a, n) ; J(a, n)=1$ from $a ; a=1$. Assume $u=x^{(i)} \in$ $\operatorname{At}\left(C_{\mathbf{E}}(\mathbf{A})\right), 0^{\prime} \geq x \in A t(\mathbf{A})$, and $0 \leq i \in \omega$. Then $x \leq 1=a ; a=\sum\{y ; z$ : $a \geq y, z \in A t(\mathbf{A})\}$ so there are atoms $a \geq y, z \in A t(\mathbf{A})$ such that $x \leq y ; z$. Note that $\mathrm{c}(y)=\mathrm{c}(z)=a$. Choose any $j$ such that $\max (i, n) \leq j \in \omega$. Then $T(i, j, j)$ holds, so $\left[x^{(i)}, y^{(j)}, z^{(j)}\right]$ is a cycle of $C_{\mathbf{E}}(\mathbf{A})$ by (6), hence $u=x^{(i)} \leq y^{(j)} ; z^{(j)} \leq$ 
$J(a, n) ; J(b, n)$. This shows $J(a, n) ; J(a, n)=1$, and we obtain $J(b, n) ; J(b, n)=$ $1=J(c, n) ; J(c, n)$ similarly from $b ; b=c ; c=1$.

This completes the proof that $J(a, n), J(b, n), J(c, n)$ is a flexible trio of $\mathbf{B}_{n}$. By Theorem 4 below, $\mathbf{B}_{n}$ has the 1-point extension property and is therefore representable. Every finitely generated subalgebra of $\mathbf{B}$ is representable, hence $\mathbf{B}$ is representable since RRA is a variety. Thus parts (i) and (ii) hold.

For part (iii)] assume such that $u ; u \cdot u=0$ whenever $1^{\prime} \neq u \in A t(\mathbf{A})$. Suppose that $\rho$ is a complete representation of $\mathbf{B}$. By definition, a complete representation preserves all joins. In particular, the join of the diversity atoms is 0 ', so the union of the representations of the diveristy atoms must be the diversity relation, consisting of all pairs of distinct objects. Thus every pair $\langle i, j\rangle$ with $i \neq j$ is in the representation of a diversity atom of $\mathbf{B}$. But $\mathbf{A} \cong \mathbf{A}^{\prime} \subseteq \mathbf{B}$ via $a \mapsto J(a, 0)$, so the representation $\rho$ determines a coloring of the edges of $K_{\omega}$ (the complete graph on countably many vertices) as follows: the "color" of the edge $(i, j)$ is $a \in A t(\mathbf{A})$ if $\langle i, j\rangle \in \rho(J(a, 0))$. This coloring has no monochrome triangles because $\mathbf{A}$ has no 1-cycles, contrary to Berge [3, Prop. 1, p. 440]. Therefore there is no such $\rho$ and B is not completely representable.

Corollary 1. If $\mathbf{A}$ is a finite Monk algebra with six or more colors then $\mathbf{A}$ is in the class $K$ defined in Problem 1. In fact, $\mathbf{A}$ is a subalgebra of the completion of a relation algebra $\mathbf{B}$ such that

- $\mathbf{B}$ is a countable, atomic, symmetric, integral relation algebra that is generated by its atoms,

- every finitely generated subalgebra of $\mathbf{B}$ is contained in a finite subalgebra of $\mathbf{B}$ with the 1-point extension property,

- the completion of $\mathbf{B}$ has the same atom structure as $\mathbf{B}$, is isomorphic to the complex algebra of the atom structure of $\mathbf{B}$, and is not representable,

- $\mathbf{B}$ is representable but not completely representable.

The smallest example to which these considerations apply is $\mathbf{A}=\mathbf{E}_{7}^{23}$, the example considered earlier. This algebra is a Monk algebra with six colors and no 1-cycles, obtained from itself by splitting. By the Street-Whitehead-Comer method, $\mathbf{E}_{7}^{23} \in$ RRA because $\mathbf{E}_{7}^{23}$ has square representations on sets containing 97,157 , and 277 elements. [AMS Meeting, Iowa City, March 2011.] Suppose the diversity atoms of $\mathbf{E}_{7}^{23}$ are $\mathbf{e}_{1}, \ldots, \mathbf{e}_{6}$. Let $a_{1}=\mathbf{e}_{1}+\mathbf{e}_{2}, a_{2}=\mathbf{e}_{3}+\mathbf{e}_{4}$, and $a_{3}=\mathbf{e}_{5}+\mathbf{e}_{6}$. Then $\mathbf{A}$ is special extension of the subalgebra $\mathbf{E}$ whose atoms are $1^{\prime}, a_{1}, a_{2}, a_{3}$, and $a_{1}, a_{2}, a_{3}$ is a flexible trio of individually flexible atoms in $\mathbf{E}$. Then RRA is not closed under completions because $C_{\mathbf{E}}\left(\mathbf{E}_{7}^{23}\right)$ is the non-representable completion of the atomic representable subalgebra of $C_{\mathbf{E}}\left(\mathbf{E}_{7}^{23}\right)$ generated by $A t\left(C_{\mathbf{E}}\left(\mathbf{E}_{7}^{23}\right)\right)$. In the next section we compute the exact degree of non-representability of $C_{\mathbf{E}}\left(\mathbf{E}_{7}^{23}\right)$.

\section{Cylindric algebras}

$\mathrm{CA}_{n}$ is the class of $n$-dimensional cylindric algebras. Given a cylindric algebra $\mathbf{D} \in \mathrm{CA}_{n}$ of dimension $n \geq 3$, the relation algebraic reduct $\mathbf{R a}(\mathbf{D})$ is defined in [8, Def. 5.3.7] and is a relation algebra if $n \geq 4$ by [8, Def. 5.3.8]. For any class $K \subseteq \mathrm{CA}_{n}$ with $3 \leq n$, let $\mathrm{Ra} K$ be the class of relation-algebraic reducts of subalgebras of neat 3 -dimensional reducts of algebras in $K$ :

$$
\mathrm{Ra} K=\mathbf{R a}^{*} \mathbf{S N r}_{3} K
$$


By [8, 5.3.9, 5.3.16, 5.3.17], we have

$$
\mathrm{RRA}=\prod_{n \in \omega} \mathrm{RaCA}_{n+4} \subseteq \cdots \subseteq \mathrm{RaCA}_{5} \subseteq \mathrm{RaCA}_{4}=\mathrm{RA} .
$$

Every non-representable relation algebra lies somewhere on this chain. The location of the example $C_{\mathbf{E}}\left(\mathbf{E}_{7}^{23}\right)$ is determined by the main result in this section, which implies

$$
C_{\mathbf{E}}\left(\mathbf{E}_{7}^{23}\right) \in \mathrm{RaCA}_{7} \sim \mathrm{RaCA}_{8} .
$$

Definition 9. Assume $\mathbf{A} \in \mathrm{NA}$ is atomic and $k \leq \omega$. Two basic matrices $\mu$ and $\mu^{\prime}$ in $B_{k}(\mathbf{A})$ agree up to $i$ if $\mu_{l, m}=\mu_{l, m}^{\prime}$ whenever $i \neq l, m \in k$, and they agree up to $i, j$ if $\mu_{l, m}=\mu_{l, m}^{\prime}$ whenever $i, j \neq l, m \in k$. We say that $\mathcal{M} \subseteq B_{k}(\mathbf{A})$ is an $k$-dimensional relational basis for $\mathbf{A}$ if

$\left(\mathrm{R}_{0}\right)$ for every atom $a \in A t(\mathbf{A})$ there is a basic matrix $\mu \in \mathcal{M}$ such that $\mu_{0,1}=a$,

$\left(\mathrm{R}_{1}\right)$ if $\mu \in \mathcal{M}, i, j<k, x, y \in$ At $\mathbf{A}, \mu_{i, j} \leq x ; y$, and $i, j \neq l<k$, then there is some $\mu^{\prime} \in \mathcal{M}$ such that $\mu$ and $\mu^{\prime}$ agree up to $l, \mu_{i, l}^{\prime}=x$, and $\mu_{l, j}^{\prime}=y$.

For any $i, j<k$ let

$$
\begin{aligned}
T_{i}^{k}(\mathbf{A}) & =\left\{\left\langle\mu, \mu^{\prime}\right\rangle \in B_{k}(\mathbf{A}) \times B_{k}(\mathbf{A}): \mu \text { and } \mu^{\prime} \text { agree up to } i\right\}, \\
E_{i, j}^{k}(\mathbf{A}) & =\left\{\mu \in B_{k}(\mathbf{A}): \mu_{i, j} \leq 1^{\prime}\right\} .
\end{aligned}
$$

We say that $\mathcal{M} \subseteq B_{k} \mathbf{A}$ is a $k$-dimensional cylindric basis for $\mathbf{A}$ if

$\left(\mathrm{C}_{0}\right)$ if $a, b, c \in A t(\mathbf{A})$, and $a \leq b ; c$, then there is a basic matrix $\mu \in \mathcal{M}$ such that $\mu_{01}=a, \mu_{02}=b$, and $\mu_{21}=c$,

$\left(\mathrm{C}_{1}\right)$ if $\mu, \mu^{\prime} \in \mathcal{M}, i, j<k, i \neq j$, and $\mu$ agrees with $\mu^{\prime}$ up to $i, j$, then there is some $\mu^{\prime \prime} \in \mathcal{M}$ such that $\mu^{\prime \prime}$ agrees with $\mu$ up to $i$, and $\mu^{\prime \prime}$ agrees with $\mu^{\prime}$ up to $j$, i.e., $\left\langle\mu^{\prime \prime}, \mu\right\rangle \in T_{i}^{k}(\mathbf{A})$ and $\left\langle\mu^{\prime \prime}, \mu^{\prime}\right\rangle \in T_{j}^{k}(\mathbf{A})$,

$\left(\mathrm{C}_{2}\right)$ if $\mu \in \mathcal{M}$ and $i, j<k$ then $\mu[i / j] \in \mathcal{M}$, where $[i / j](m)=m$ if $i \neq m<k$, and $[i / j](i)=j$.

For every $\mathcal{M} \subseteq B_{k}(\mathbf{A})$, let

$$
\mathbf{C a}(\mathcal{M})=\mathbf{C m}\left(\left\langle\mathcal{M}, T_{i}, E_{i j}\right\rangle_{i, j<k}\right)
$$

be the complex algebra of the relational structure $\left\langle\mathcal{M}, T_{i}, E_{i j}\right\rangle_{i, j<k}$, as defined in 7 , 2.7.33], where $E_{i j}=E_{i j}^{k}(\mathbf{A}) \cap \mathcal{M}$ and $T_{i}=T_{i}^{k}(\mathbf{A}) \cap(\mathcal{M} \times \mathcal{M})$ for all $i, j<k$.

Theorem 3. Assume $4 \leq r \in \omega, \mathbf{A}=\mathbf{E}_{r+3}^{23}$, and the atoms of $\mathbf{A}$ are

$$
\mathbf{e}_{0}=1^{\prime}, \mathbf{e}_{1}, \mathbf{e}_{2}, \mathbf{e}_{3}, \mathbf{e}_{4}, \mathbf{e}_{5}, \mathbf{e}_{6}, \mathbf{e}_{7}, \cdots, \mathbf{e}_{r+2} .
$$

Then $\mathbf{A}$ is a special extension of a subalgebra $\mathbf{E}$ whose $r$ atoms are

$$
\begin{aligned}
\mathrm{a}_{0}=\mathbf{e}_{0}=1 & \mathrm{a}_{1}=\mathbf{e}_{1}+\mathbf{e}_{2} & \mathrm{a}_{2}=\mathbf{e}_{3}+\mathbf{e}_{4} & \mathrm{a}_{3} & =\mathbf{e}_{5}+\mathbf{e}_{6} \\
\mathrm{a}_{4} & =\mathbf{e}_{7} & \ldots & \mathrm{a}_{r-1} & =\mathbf{e}_{r+2}
\end{aligned}
$$

and $\mathrm{a}_{1}, \mathrm{a}_{2}, \mathrm{a}_{3}$ is a flexible trio of $\mathbf{E}$, so by Ths.1,2, the atom-generated subalgebra of the complete atomic relation algebra $C_{\mathbf{E}}(\mathbf{A})$ is an atomic atom-generated symmetric integral representable relation algebra with finite finitely-generated subalgebras, and if $3 \leq n \leq r+3$ then

(i) $B_{n}\left(C_{\mathbf{E}}(\mathbf{A})\right)$ is an n-dimensional cylindric basis for $C_{\mathbf{E}}(\mathbf{A})$,

(ii) $\mathbf{C a}\left(B_{n}\left(C_{\mathbf{E}}(\mathbf{A})\right)\right)$ is a complete atomic $n$-dimensional cylindric algebra, 
(iii) $C_{\mathbf{E}}(\mathbf{A})$ is isomorphic to the relation algebraic reduct of $\mathbf{C a}\left(B_{n}\left(C_{\mathbf{E}}(\mathbf{A})\right)\right)$ and $C_{\mathbf{E}}(\mathbf{A}) \in \mathrm{RaCA}_{n}$,

(iv) $\mathbf{C a}\left(B_{n}\left(C_{\mathbf{E}}(\mathbf{A})\right)\right) \notin \mathbf{S N r}{ }_{n} \mathrm{CA}_{r+4}$.

Proof. By [23, Th. 7], in order to prove $B_{n}\left(C_{\mathbf{E}}(\mathbf{A})\right)$ is a cylindric basis for $C_{\mathbf{E}}(\mathbf{A})$ it is enough to show, given $n-2$ pairs of diversity atoms $u_{1}, v_{1}, \cdots, u_{n-2}, v_{n-2}$ of $C_{\mathbf{E}}(\mathbf{A})$, that $\prod_{1 \leq i \leq n-2} u_{i} ; v_{i} \neq 0$. We will find a diversity atom $w$, such that $w$ is included in every product $u_{i} ; v_{i}, 1 \leq i \leq n-2$. Any product $u_{i} ; v_{i}$ that is equal to 0 ' or 1 imposes no restriction on our choice of $w$. We therefore assume that none of the products is $0^{\prime}$ or 1 , i.e., $0^{\prime} \neq u_{i} ; v_{i} \neq 1$ whenever $1 \leq i \leq n-2$.

Consequently, for every product $u_{i} ; v_{i}$ we know that there cannot be distinct atoms $a, b \in A t(\mathbf{E})$ such that $u_{i} \leq J(a, 0)$ and $v_{i} \leq J(b, 0)$, because we would obtain $u_{i} ; v_{i}=J(a ; b, 0)$ from $a \neq b$ by Lemma 3 , and a computation in $\mathbf{E}$ shows $a ; b=0$ ' since $a \neq b$, forcing $u_{i} ; v_{i}=0$ ' in $C_{\mathbf{E}}(\mathbf{A})$, contrary to our assumption that no product is 0 ' or 1 . Therefore, there is a function $f:\{1, \cdots, n-2\} \rightarrow\{1, \cdots, r-1\}$ such that

$$
u_{i}+v_{i} \leq J\left(\mathrm{a}_{f(i)}, 0\right) \text { for all } i \in\{1, \cdots, n-2\} .
$$

We see next that every index in $\{1, \cdots, r-1\}$ is in the range of $f$. Suppose some index $j\{1, \cdots, r-1\}$ is not in the range of $f$. Consider any product $u_{i} ; v_{i}$ with $1 \leq i \leq n-2$. Let $k=f(i)$ and note that $k \neq j$. There are atoms $x, y \in A t(\mathbf{A})$ such that

$$
x+y \leq \mathrm{a}_{k}=\mathrm{c}(x)=\mathrm{c}(y), \quad u_{i} \leq J(x, 0), \quad v_{i} \leq J(y, 0) .
$$

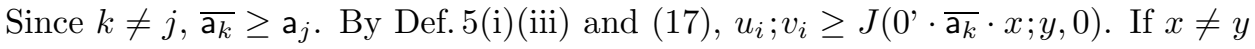
then $x ; y=0$ ' in $\mathbf{A}$, so

$$
u_{i} ; v_{i} \geq J\left(0^{\prime} \cdot \overline{\mathrm{a}_{k}} \cdot x ; y, 0\right)=J\left(0^{\prime} \cdot \overline{\mathrm{a}_{k}}, 0\right) \geq J\left(\mathrm{a}_{j}, 0\right) .
$$

If $x=y$ then $x ; y=\bar{x} \geq \overline{\mathrm{a}_{k}}$ in $\mathbf{A}$, so $\overline{\mathrm{a}_{k}} \cdot x ; y=\overline{\mathrm{a}_{k}}$, and again we have (18) 4 Since (18) holds for every $i$, we obtain much more than $\prod_{1=i}^{n-2} u_{i} ; v_{i} \neq 0$, in fact,

$$
0 \neq J\left(\mathrm{a}_{j}, 0\right) \leq \prod_{i=1}^{n-2} u_{i} ; v_{i} .
$$

Therefore, assume that $f$ is surjective. Next we show that $f$ is actually maps two distinct indices onto each of the atoms $\mathrm{a}_{1}, \mathrm{a}_{2}$, and $\mathrm{a}_{3}$, i.e., those atoms of $\mathbf{E}$ that are the join of two atoms of $\mathbf{A}$. We prove this only for $\mathrm{a}_{1}$. Since 1 is in the range of $f$, we'll suppose, for specificity and simplicity of notation, that $1=f(1)$, i.e., $u_{1}+v_{1} \leq J\left(\mathrm{a}_{1}, 0\right)$. We wish to show that $1=f(i)$ for some $i \neq 1$, so we assume this does not happen, i.e., assume $1 \neq f(i)$ for all $i \in\{2, \cdots, n-2\}$. Now $\mathrm{a}_{1}=\mathbf{e}_{1}+\mathbf{e}_{2}$, so there are atoms $x, y \in\left\{\mathbf{e}_{1}, \mathbf{e}_{2}\right\}$ and indices $k, l \in \omega$ such that $x+y \leq \mathrm{a}_{1}$, $u_{1}=x^{(k)}$, and $v_{1}=y^{(l)}$. Let $m=\max (k, l)$. Notice that $T(k, l, m)$ holds and $\mathbf{E}_{r+3}^{23}$ contains the 2-cycles $\left[\mathbf{e}_{1}, \mathbf{e}_{1}, \mathbf{e}_{2}\right]$ and $\left[\mathbf{e}_{1}, \mathbf{e}_{2}, \mathbf{e}_{2}\right]$. It follows by (6) that

$$
\begin{aligned}
\mathrm{e}_{1}^{(k)} ; \mathrm{e}_{1}^{(l)} \geq \mathrm{e}_{2}^{(m)} \\
\mathrm{e}_{1}^{(k)} ; \mathrm{e}_{2}^{(l)}=\mathrm{e}_{2}^{(k)} ; \mathrm{e}_{1}^{(l)} \geq \mathrm{e}_{1}^{(m)}+\mathrm{e}_{2}^{(m)} \\
\mathrm{e}_{2}^{(k)} ; \mathrm{e}_{2}^{(l)} \geq \mathrm{e}_{1}^{(m)}
\end{aligned}
$$

${ }^{4}$ By the way, we've shown $j \neq f(i) \Longrightarrow u_{i} ; v_{i} \geq J\left(\mathrm{a}_{j}, 0\right)$. 
ROGER D. MADDUX

We may therefore let $w=\mathrm{e}_{1}^{(m)}$ if $x=y=\mathbf{e}_{1}, w=\mathrm{e}_{2}^{(m)}$ if $x=y=\mathbf{e}_{2}$, and either $w=\mathbf{e}_{1}$ or $w=\mathbf{e}_{2}$ if $x \neq y$. In every case, $u_{1} ; v_{1} \geq w$. For products other than $u_{1} ; v_{1}$, note that if $2 \leq i \leq n-2$, then by our assumption we have $1 \neq f(i)$, hence by the footnote, $u_{i} ; v_{i} \geq J\left(\mathrm{a}_{1}, 0\right) \geq w$. This shows $w \leq \prod_{i=2}^{n-2} u_{i} ; v_{i}$, which, together with $w \leq u_{1} ; v_{1}$, gives us $w \leq \prod_{i=1}^{n-2} u_{i} ; v_{i}$.

At this point we know that either we are done because we have proved $\prod_{i=1}^{n-2} u_{i} ; v_{i} \neq$ 0 , or else $f$ maps at least one index from $\{1, \cdots, n-2\}$ onto each of the indices in $\{4, \cdots, r-1\}$, and $f$ maps at least two indices from $\{1, \cdots, n-2\}$ onto each of the indices in $\{1,2,3\}$, But $|\{1, \cdots, n-2\}|=n-2,|\{4, \cdots, r-1\}|=r-4$, and $|\{1,2,3\}|=3$, so we must have $n-2 \geq r-4+2 \cdot 3=r+2$, but our restriction on $r$ is $n \leq r+3$, so $n-2 \leq r+1$, a contradiction. Therefore we do in fact know that $0 \neq \prod_{i=1}^{n-2} u_{i} ; v_{i}$, as desired. This shows $B_{n}\left(C_{\mathbf{E}}(\mathbf{A})\right)$ is a cylindric basis for $C_{\mathbf{E}}(\mathbf{A})$ and completes the proof of part (i) Parts (ii) and (iii) follow from part (i) by [23, Theorem 10].

For part (iv), assume to the contrary that $\mathbf{C a}\left(B_{n}\left(C_{\mathbf{E}}(\mathbf{A})\right)\right) \subseteq \mathbf{N r}_{n} \mathbf{D}$ for some $\mathbf{D} \in \mathrm{CA}_{r+4}$. We get a contradiction by finding a subalgebra $\mathbf{F}$ of $\mathbf{C a}\left(B_{n}\left(C_{\mathbf{E}}(\mathbf{A})\right)\right)$ which is not in $\mathrm{SNr}_{n} \mathrm{CA}_{r+4}$. From Theorem 5 with $p=r+3$ we get

$$
\mathbf{C a}\left(B_{3}(\mathbf{F})\right) \notin \mathbf{S N r}{ }_{3} \mathrm{CA}_{p+1}=\mathbf{S N r}_{3} \mathrm{CA}_{r+4}
$$

For this we choose an arbitrary finite parameter $N \in \omega$ and make it big enough. For this fixed $N$ there is a finite subalgebra $\mathbf{F}$ of $C_{\mathbf{E}}(\mathbf{A})$ whose atoms are 1', $\mathrm{e}_{i}^{(j)}$ and $J\left(\mathbf{e}_{i}, N\right)$ for $1 \leq i \leq r+2$ and $j<N$. This finite subalgebra $\mathbf{F}$ has a subalgebra isomorphic to $\mathbf{E}_{r+3}^{23}$, whose atoms are 1' and $J\left(\mathbf{e}_{i}, 0\right)$ for $1 \leq i \leq r+2$. By Theorem 5 in the next section, a finite extension of $\mathbf{E}_{r+3}^{23}$ with enough atoms satisfies (20). By choosing $N$ large enough, the extension $\mathbf{F}$ of $\mathbf{E}_{r+3}^{23}$ has enough atoms.

\section{REFERENCES}

[1] H. Andréka, R. D. Maddux, and I. Németi, Splitting in relation algebras, Proc. Amer. Math. Soc. 111 (1991), no. 4, 1085-1093. MR 1052567 (91g:03126)

[2] Hajnal Andréka, István Németi, and Tarek Sayed Ahmed, Omitting types for finite variable fragments and complete representations of algebras, J. Symbolic Logic 73 (2008), no. 1, 65-89. MR 2387933 (2008m:03130)

[3] Claude Berge, Graphs and hypergraphs, North-Holland Publishing Co., Amsterdam, 1973, Translated from the French by Edward Minieka, North-Holland Mathematical Library, Vol. 6. MR 0357172 (50 \#9640)

[4] Stephen D. Comer, Color schemes forbidding monochrome triangles, Proceedings of the fourteenth Southeastern conference on combinatorics, graph theory and computing (Boca Raton, Fla., 1983), vol. 39, 1983, pp. 231-236. MR 734546 (85f:05052)

[5] Combinatorial aspects of relations, Algebra Universalis 18 (1984), no. 1, 77-94.

[6] M. F. Frias and R. D. Maddux, Non-embeddable simple relation algebras, Algebra Universalis 38 (1997), no. 2, 115-135. MR 1608984 (99a:03063)

[7] Leon Henkin, J. Donald Monk, and Alfred Tarski, Cylindric algebras. Part I. With an introductory chapter: General theory of algebras, North-Holland Publishing Co., Amsterdam, 1971, Studies in Logic and the Foundations of Mathematics, Vol. 64. MR 0314620 (47 \#3171)

[8] Cylindric algebras. Part II, Studies in Logic and the Foundations of Mathematics, vol. 115, North-Holland Publishing Co., Amsterdam, 1985. MR 781930 (86m:03095b)

[9] Robin Hirsch, Completely representable relation algebras, Bull. IGPL 3 (1995), no. 1, 77-91. MR 1330986 (96d:03082)

[10] Robin Hirsch and Ian Hodkinson, Complete representations in algebraic logic, J. Symbolic Logic 62 (1997), no. 3, 816-847. MR 1472125 (98m:03123) 
[11] Relation Algebras by Games, Studies in Logic and the Foundations of Mathematics, vol. 147, North-Holland Publishing Co., Amsterdam, 2002, With a foreword by Wilfrid Hodges. MR 1935083 (2003m:03001)

[12] - Strongly representable atom structures of relation algebras, Proc. Amer. Math. Soc. 130 (2002), no. 6, 1819-1831. MR 1887031 (2002k:03113)

[13] _ Strongly representable atom structures of cylindric algebras, J. Symbolic Logic $\mathbf{7 4}$ (2009), no. 3, 811-828. MR 2548463 (2011c:03147)

[14] Robin Hirsch, Ian Hodkinson, and Roger D. Maddux, Provability with finitely many variables, Bull. Symbolic Logic 8 (2002), no. 3, 348-379. MR 1931348 (2003m:03013)

[15] Ian Hodkinson, Atom structures of cylindric algebras and relation algebras, Ann. Pure Appl. Logic 89 (1997), no. 2-3, 117-148. MR 1490103 (99c:03103)

[16] Ian Hodkinson and Yde Venema, Canonical varieties with no canonical axiomatisation, Trans. Amer. Math. Soc. 357 (2005), no. 11, 4579-4605. MR 2156722 (2006e:03106)

[17] Mohamed Khaled and Tarek Sayed Ahmed, Classes of algebras that are not closed under completions, Bull. Sect. Logic Univ. Lódź 38 (2009), no. 1-2, 29-43. MR 2605423 (2011a:03071)

[18] - On complete representations of algebras of logic, Log. J. IGPL 17 (2009), no. 3, 267-272. MR 2507303 (2011a:03072)

[19] Roger C. Lyndon, The representation of relational algebras, Ann. of Math. (2) 51 (1950), 707-729. MR 0037278 (12,237a)

[20] Roger Maddux, Some varieties containing relation algebras, Trans. Amer. Math. Soc. 272 (1982), no. 2, 501-526. MR 662049 (84a:03079)

[21] Roger D. Maddux, Topics in relation algebras, Ph.D. thesis, University of California, Berkeley, 1978, pp. iii+241.

[22] _ Finite integral relation algebras, Universal Algebra and Lattice Theory (Charleston, S.C., 1984), Springer, Berlin, 1985, pp. 175-197.

[23] _ Nonfinite axiomatizability results for cylindric and relation algebras, J. Symbolic Logic 54 (1989), no. 3, 951-974. MR 1011183 (90f:03099)

[24] _ Relation Algebras, Studies in Logic and the Foundations of Mathematics, vol. 150, Elsevier B. V., Amsterdam, 2006. MR 2269199 (2007j:03096)

[25] J. Donald Monk, Nonfinitizability of classes of representable cylindric algebras, J. Symbolic Logic 34 (1969), 331-343. MR 0256861 (41 \#1517)

[26] _ Completions of Boolean algebras with operators, Math. Nachr. 46 (1970), 47-55. MR 0277369 (43 \#3102)

[27] Tarek Sayed Ahmed, Neat embedding is not sufficient for complete representability, Bull. Sect. Logic Univ. Lódź 36 (2007), no. 1-2, 29-35. MR 2357188 (2008g:03103)

[28] - A note on atom structures of relation and cylindric algebras, Int. J. Algebra 2 (2008), no. 9-12, 595-601. MR 2443834 (2009g:03101)

[29] - A note on atom structures of relation and cylindric algebras, Bull. Sect. Logic Univ. Lódź 37 (2008), no. 1, 29-35. MR 2441102 (2009g:03100)

[30] _ Weakly representable atom structures that are not strongly representable, with an application to first order logic, MLQ Math. Log. Q. 54 (2008), no. 3, 294-306. MR 2417802 (2009d:03161)

[31] _ A simple construction of representable relation algebras with non-representable completions, MLQ Math. Log. Q. 55 (2009), no. 3, 237-244. MR 2519240 (2010i:03072)

[32] Tarek Sayed Ahmed and Basim Samir, The class $\mathbf{S N r}_{3} \mathbf{C A} \mathbf{A}_{k}$ is not closed under completions, Log. J. IGPL 16 (2008), no. 5, 427-429. MR 2453362

[33] Alfred Tarski, Contributions to the theory of models. III, Nederl. Akad. Wetensch. Proc. Ser. A. 58 (1955), 56-64 = Indagationes Math. 17, 56-64 (1955). MR 0066303 (16,554h)

\section{ApPEndix A. A representation result}

The next theorem was invoked in the proof of Theorem 2

Theorem 4. Assume $\mathbf{A}$ is an atomic symmetric integral relation algebra containing a flexible trio. Then $\mathbf{A}$ has the 1-point extension property and $\mathbf{A} \in \mathrm{RRA}$.

Proof. Once it has been shown that $\mathbf{A}$ has the 1-point extension property, it follows from some additional observations about the behavior of identity elements that the 
set $B_{k}(\mathbf{A})$ of basic $k$-by- $k$ matrices of atoms of $\mathbf{A}$ is a relational basis for $\mathbf{A}$ whenever $k \geq 3$ (see Def. 91). Then $\mathbf{A} \in \mathrm{RA}_{k}$ for all $k \geq 3$ because $\mathbf{A}$ is atomic and has a $k$-dimensional relational basis, hence $\mathbf{A} \in \bigcap_{k \geq 3} \mathrm{RA}_{k}=\mathrm{RRA}$. In fact, when $\mathbf{A}$ is atomic (as in the proof of Theorem 11) it is easy to prove directly from the 1-point extension property that $\mathbf{A}$ has a complete representation on $\omega$.

We show next that there is a function $f$ such that for any diversity atoms $x$ and $y$, we have $f(x, y) \in\{a, b, c\}$ and

$$
x ; f(x, y)=y ; f(x, y) \geq 0,
$$

For an arbitrary diversity atom $x$, consider the set

$$
Z_{x}:=\left\{z: x ; z \geq 0^{\prime}, 0^{\prime} \geq z \in \operatorname{At}(\mathbf{A})\right\} .
$$

If $x \in\{a, b, c\}$ then $a, b, c \in Z_{x}$ by (12). If $x \notin\{a, b, c\}$ then by (13), $\{a, b, c\} \cap Z_{x}$ has at least two elements. Consequently, if $y$ is another, possibly different, diversity atom of $\mathbf{A}$, then, since $Z_{x}$ and $Z_{y}$ are subsets of the 3 -element set $\{a, b, c\}$ and they each contain at least two elements, they must intersect. We choose a value in the intersection as $f(x, y)$. There are several ways to do this. We pick this one-

(A) if $a \in Z_{x} \cap Z_{y}$ then $f(x, y)=a$,

(B) if $a \notin Z_{x} \cap Z_{y}$ and $b \in Z_{x} \cap Z_{y}$ then $f(x, y)=b$,

(C) if $a \notin Z_{x} \cap Z_{y}$ and $b \notin Z_{x} \cap Z_{y}$ then $f(x, y)=c$.

It is obvious from (22) that (21) holds in the first two cases. We need to show (21) also holds in the third case (C), i.e., that under the assumptions $a \notin Z_{x} \cap Z_{y}$ and $b \notin Z_{x} \cap Z_{y}$ we have $c \in Z_{x} \cap Z_{y}$. But if $c \notin Z_{x} \cap Z_{y}$ then we would conclude that $Z_{x} \cap Z_{y}$ is empty, since it is a subset of $\{a, b, c\}$ that excludes each of $a, b$, and $c$ by our assumptions, contrary to the observations made above.

For the 1-point extension property, assume $k \in \omega, \mu \in B_{k}(\mathbf{A}), \mu_{l, m}$ satisfies the identity condition, $x, y$ are diversity atoms of $\mathbf{A}$, and $\mu_{i, j} \leq x ; y$ for some fixed $i, j<k$. We will prove that $\mu$ has a 1-point extension $\mu^{\prime} \in B_{k+1}(\mathbf{A})$ such that $\mu \subseteq \mu^{\prime}, \mu_{i, k}^{\prime}=x=\mu_{k, i}^{\prime}, \mu_{k, j}^{\prime}=y=\mu_{j, k}^{\prime}$, and if $k>l \neq i, j$ then

$$
\mu_{l, k}^{\prime}=\mu_{k, l}^{\prime}=f(x, y)
$$

Note that by definitions and (21) we have

$$
x ; \mu_{k, l}^{\prime}=0^{\prime}=y ; \mu_{k, l}^{\prime} .
$$

Having chosen $\mu_{k, l}^{\prime}$ to be either $a$ or $b$ or $c$, we must check for each $l<k$ whether the first two crucial cycle equations below hold, and finally whether the third equation holds for those points $l, m<k$ where $l \neq m$ and $\{l, m\} \cap\{i, j\}=\emptyset$.

$$
\begin{array}{rlrl}
\mu_{i, l} & \leq \mu_{i, k}^{\prime} ; \mu_{k, l}^{\prime} & & \text { i.e., }\left[\mu_{i, k}^{\prime}, \mu_{k, l}^{\prime}, \mu_{i, l}\right] \text { is a cycle } \\
\mu_{j, l} & \leq \mu_{j, k}^{\prime} ; \mu_{k, l}^{\prime} & \text { i.e., }\left[\mu_{j, k}^{\prime}, \mu_{k, l}^{\prime}, \mu_{j, l}\right] \text { is a cycle } \\
\mu_{l, m} \leq \mu_{l, k}^{\prime} ; \mu_{k, m}^{\prime} & \text { i.e., }\left[\mu_{l, k}^{\prime}, \mu_{k, m}^{\prime}, \mu_{l, m}\right] \text { is a cycle }
\end{array}
$$

The first two equations hold by (23) (their right sides are 0 '). For the third equation, first note that $\mu_{l, k}^{\prime}=\mu_{k, m}^{\prime}$ because the value depends only on $x, y$, not on $l$ or $m$. The right side of the third equation is therefore $a ; a$ or $b ; b$ or $c ; c$, but $a ; a=b ; b=$ $c ; c=1$, so the third equation holds. 


\section{APPENDIX B. A non-representation result}

Theorem 5. Assume

(i) $\mathbf{E} \subseteq \mathbf{A} \in \mathrm{NA}$,

(ii) $\mathbf{E}$ is finite and symmetric, $1^{\prime} \in A t(\mathbf{E})$, and $\mathbf{E}$ has $p>3$ atoms,

(iii) $\mathbf{E}$ has no 1-cycles: $u ; u \cdot u=0$ if $0^{\prime} \geq u \in A t(\mathbf{E})$,

(iv) $\mathbf{A}$ is finite and symmetric, 1' $\in A t(\mathbf{A})$, and some diversity atom of $\mathbf{E}$ is the join of at least $p^{p-1}$ atoms of $\mathbf{A}$.

Then

(i) $\mathbf{A} \notin \mathbf{S R a C A}{ }_{p+1}$,

(ii) $\mathbf{C a}\left(B_{3}(\mathbf{A})\right) \notin \mathbf{S} \mathrm{Nr}_{3} \mathrm{CA}_{p+1}$.

Proof of (i). Let the atoms of $\mathbf{E}$ be $1^{\prime}=\mathrm{a}_{0}, \mathrm{a}_{1}, \ldots, \mathrm{a}_{p-1}$, where $p \geq 3$ and $\mathrm{a}_{1}$ is a diversity atom of $\mathbf{E}$ which is the join of at least $p^{p-1}$ atoms of $\mathbf{A}$. Let $\mathrm{c}(x)$ be the atom of $\mathbf{E}$ containing $x \in A t(\mathbf{A})$. We refer to $\mathrm{c}(x)$ as the "color" of $x$ (or "cover", as in the definition of splitting).

Assume, for the sake of obtaining a contradiction, that $\mathbf{A} \subseteq \mathbf{R a}(\mathbf{D})$ for some $\mathbf{D} \in \mathbf{C A}_{p+1}$. All the elements of $\mathbf{A}$, in particular all the atoms, are 2-dimensional elements of $\mathbf{D}$, i.e.,

$$
A t(\mathbf{A}) \subseteq N r_{2} \mathbf{D} .
$$

If $1<q \leq p+1$ and $x \in D$, we say $x$ is $q$-color-ordered if $\mathrm{c}(u)=\mathrm{c}(v)$ whenever $u, v \in A t(\mathbf{A}), 0 \leq i<j<k<q$, and $x \leq \mathrm{s}_{i}^{0} \mathrm{~s}_{j}^{1} u \cdot \mathrm{s}_{i}^{0} \mathrm{~s}_{k}^{1} v$.

The element $x \in D$ is $q$-covered if there are atoms $u_{i, j} \in A t(\mathbf{A})$ for $0 \leq i<$ $j<q$ such that $x \leq \prod_{0 \leq i<j<q} \mathbf{s}_{i}^{0} \mathbf{s}_{j}^{1} u_{i, j}$, in which case the atoms $u_{i, j}$ are said to be a $q$-covering of $x$.

The atoms in a $q$-covering of a non-zero $x \in D$ are unique, for if there are further atoms $v_{i, j} \in A t(\mathbf{A}), 0 \leq i<j<q$, such that $x \leq \prod_{0 \leq i<j<q} \mathbf{s}_{i}^{0} \mathbf{s}_{j}^{1} v_{i, j}$, then, since substitution is a complete Boolean endomorphism by [7, 1.5.3], we have

$$
\begin{aligned}
0 \neq x & \leq \prod_{0 \leq i<j<q} \mathrm{~s}_{i}^{0} \mathrm{~s}_{j}^{1} u_{i, j} \cdot \prod_{0 \leq i<j<q} \mathrm{~s}_{i}^{0} \mathbf{s}_{j}^{1} v_{i, j} \\
& =\prod_{0 \leq i<j<q} \mathbf{s}_{i}^{0} \mathbf{s}_{j}^{1}\left(u_{i, j} \cdot v_{i, j}\right),
\end{aligned}
$$

but if $u_{i, j} \neq v_{i, j}$ then, since distinct atoms are disjoint, a zero occurs with a contradiction ensuing. Thus $u_{i, j}=v_{i, j}$ whenever $0 \leq i<j<q$.

We will construct by induction for each dimension from $q=2$ up to $q=p+1$ a set $S_{q} \subseteq N r_{q} \mathbf{D}$ such that

(i) $S_{q}$ has at least $p^{p+1-q}$ elements.

(ii) Every $x \in S_{q}$ is $q$-covered, $q$-color-ordered, and non-zero, and $x \leq \mathrm{s}_{j}^{1} \mathrm{a}_{1}$ for $0<j<q$.

(iii) $\mathrm{c}_{q-1} x=\mathrm{c}_{q-1} y$ if $x, y \in S_{q}$.

(iv) $\mathrm{c}(u)=\mathrm{c}(v)$ if $u, v \in A t(\mathbf{A}), x, y \in S_{q}, x \leq \mathrm{s}_{q-2}^{0} \mathrm{~s}_{q-1}^{1} u$, and $y \leq \mathrm{s}_{q-2}^{0} \mathrm{~s}_{q-1}^{1} v$.

(v) $u \neq v$ if $u, v \in$ At (A), $x, y \in S_{q}, x \leq \mathrm{s}_{q-1}^{1} u, y \leq \mathrm{s}_{q-1}^{1} v$, and $x \neq y$.

(vi) $u \neq v$ if $0<j<k<q, u, v \in A t(\mathbf{A}), x \in S_{q}$, and $x \leq \mathbf{s}_{j}^{1} u \cdot \mathbf{s}_{k}^{1} v$.

Let $S_{2}=\left\{x: \mathrm{a}_{1} \geq x \in A t(\mathbf{A})\right\}$.

Note that $S_{2} \subseteq N r_{2} \mathbf{D}$ by (24). Obviously $S_{2}$ has property (i) since there are at least $p^{p-1}$ atoms below $\mathrm{a}_{1}$. Let $x \in S_{2}$. Then $x$ is 2 -covered by itself (take $u_{0,1}=x$ ), 
$x$ is 2-color-ordered because the hypotheses in the definition of color-ordered are never met $(q=2$ is too small), and $x$ is not zero because it is an atom of $\mathbf{A}$. For the last part of property (ii), note that if $0 \leq i<j<q=2$ then $j=1$, and $x \leq \mathrm{a}_{1}$ by the definition of $S_{2}$, so $x \leq \mathrm{a}_{1}=\mathrm{s}_{1}^{1} \mathrm{a}_{1}=\mathrm{s}_{j}^{1} \mathrm{a}_{1}$. Therefore $S_{2}$ has property (ii). Since $\mathbf{A}$ is integral and $x \in S_{2}$ is non-zero, we have $x ; 1=1$, so

$$
\begin{aligned}
1=x ; 1 & =\mathrm{c}_{2}\left(\mathrm{~s}_{2}^{1} x \cdot \mathrm{s}_{2}^{0} 1\right) & & \text { definition of ; in } \mathbf{R a}(\mathbf{D}) \\
& =\mathrm{c}_{2} \mathrm{~s}_{2}^{1} x & & \text { [7, } 1.5 .3] \\
& =\mathrm{c}_{1} \mathrm{~s}_{1}^{2} x & & \text { [7, } 1.5 .9(\mathrm{i})] \\
& =\mathrm{c}_{1} x & & \text { [7, } 1.5 .8(\mathrm{i})], \mathrm{c}_{2} x=x
\end{aligned}
$$

It follows that property (iii) holds for $S_{2}$. For property (iv), note that since $q=2$, $\mathbf{s}_{q-2}^{0} \mathbf{s}_{q-1}^{1}$ is the identity mapping, hence the hypotheses are $u, v \in A t(\mathbf{A}), x, y \in S_{2}$, $x \leq u$, and $y \leq v$, which imply $x=u$ and $y=v$ since $u, v, x, y$ are atoms. We wish to show c $(u)=\mathrm{c}(v)$, i.e., $\mathrm{c}(x)=\mathrm{c}(y)$, but this is true by the definition of $S_{2}$. Since $q=2$, the substitution $\mathbf{s}_{q-1}^{1}$ is the identity mapping, hence the hypotheses of property (v) are $u, v \in A t(\mathbf{A}), x, y \in S_{q}, x \leq u, y \leq v$, and $x \neq y$. But these hypotheses imply $u=x \neq y=v$, so the conclusion holds trivially. Thus $S_{2}$ has property (v). Finally, $S_{2}$ has property (vi) because the hypotheses cannot hold when $q=2$.

Suppose we have a set $S_{q}$ such that $q \geq 2$ and (i) (vi). Choose an arbitrary but fixed $w \in S_{q}$, and let $S_{q}^{w}:=S_{q} \sim\{w\}$. We will obtain a function $h$ that sends every $x \in S_{q}^{w}$ to a $(q+1)$-dimensional element $h(x) \in N r_{q+1} \mathbf{D}$, and will choose $S_{q+1}$ to be a subset of the range of $h$.

For every $x \in S_{q}^{w}$, we have

$$
\begin{aligned}
0 & \neq w & & \text { (ii) } \\
& =w \cdot \mathrm{c}_{q-1} w & & {\left[7,1.1 .1\left(\mathrm{C}_{2}\right)\right] } \\
& =w \cdot \mathrm{c}_{q-1} x & & \text { (iii) } \\
& =w \cdot \mathrm{c}_{q-1} \mathrm{~s}_{q-1}^{q} x & & {[7,1.5 .8(\mathrm{i})], \mathrm{c}_{q} x=x } \\
& =w \cdot \mathrm{c}_{q} \mathrm{~s}_{q}^{q-1} x & & {[7,1.5 .9(\mathrm{i})] } \\
& =\mathrm{c}_{q}\left(w \cdot \mathrm{s}_{q}^{q-1} x\right) & & {\left[7,1.1 .1\left(\mathrm{C}_{3}\right)\right], \mathrm{c}_{q} w=w } \\
& =\mathrm{c}_{q}\left(w \cdot \mathrm{s}_{q}^{q-1} x \cdot \mathrm{s}_{q-1}^{0} \mathrm{~s}_{q}^{1}(1)\right) & & \text { A is finite } \\
& =\mathrm{c}_{q}\left(w \cdot \mathrm{s}_{q}^{q-1} x \cdot \mathrm{s}_{q-1}^{0} \mathrm{~s}_{q}^{1}\left(\sum_{y \in A t(\mathbf{A})} y\right)\right) & & {[7,1.5 .3,1.2 .6] } \\
& =\sum_{y \in A t(\mathbf{A})} \mathrm{c}_{q}\left(w \cdot \mathrm{s}_{q}^{q-1} x \cdot \mathrm{s}_{q-1}^{0} \mathrm{~s}_{q}^{1}(y)\right) & &
\end{aligned}
$$

The distributive law holds in all Boolean algebras whenever all the joins and meets involved are finite, so

$$
0 \neq w=\prod_{x \in S_{q}^{w}}\left(\sum_{y \in A t(\mathbf{A})} \mathrm{c}_{q}\left(w \cdot \mathbf{s}_{q}^{q-1} x \cdot \mathbf{s}_{q-1}^{0} \mathbf{s}_{q}^{1}(y)\right)\right)
$$




$$
=\sum_{f: S_{q}^{w} \rightarrow A t(\mathbf{A})}\left(\prod_{x \in S_{q}^{w}} \mathrm{c}_{q}\left(w \cdot \mathrm{s}_{q}^{q-1} x \cdot \mathrm{s}_{q-1}^{0} \mathrm{~s}_{q}^{1}(f(x))\right)\right) .
$$

Consequently there must be some function $f: S_{q}^{w} \rightarrow A t(\mathbf{A})$ such that

$$
0 \neq \prod_{x \in S_{q}^{w}} \mathrm{c}_{q}\left(w \cdot \mathrm{s}_{q}^{q-1} x \cdot \mathrm{s}_{q-1}^{0} \mathrm{~s}_{q}^{1}(f(x))\right) .
$$

Let $f$ be such a function. From our chosen $f$ we define additional functions $g, h$ : $S_{q}^{w} \rightarrow D$ and an element $z \in D$ as follows.

$$
\begin{aligned}
g(x) & =w \cdot \mathrm{s}_{q}^{q-1} x \cdot \mathrm{s}_{q-1}^{0} \mathrm{~s}_{q}^{1}(f(x)) & & \text { for all } x \in S_{q}^{w} \\
z & =\prod_{x \in S_{q}^{w}} \mathrm{c}_{q}(g(x)) & & \\
h(x) & =g(x) \cdot z & & \text { for all } x \in S_{q}^{w}
\end{aligned}
$$

Let $R=\left\{h(x): x \in S_{q}^{w}\right\}$. We will show that $R$ itself has properties (ii), (iii), (v), and (vi). Consequently every subset of $R$ also has these properties. We will partition $R$ into disjoint subsets that have property (iv) and prove that at least one of them must be large enough to also have property (i). We take $S_{q+1}$ to be any such subset of $R$.

To see that $R$ has property (iii), we observe that $\mathrm{c}_{q} h(x)=\mathrm{c}_{q} h(y)$ for all $x, y \in S_{q}^{w}$, because

$$
\begin{aligned}
\mathrm{c}_{q} h(x) & =\mathrm{c}_{q}(g(x) \cdot z) & & \text { (28) } \\
& =\mathrm{c}_{q}(g(x)) \cdot z & & \text { [7, } \left.1.1 .1\left(\mathrm{C}_{3}\right)\right], \mathrm{c}_{q} z=z \\
& =z & & \text { (27) }
\end{aligned}
$$

It follows that $h(x) \neq 0$ for every $x \in S_{q}$, since $z \neq 0$ by (25). This is part of property (ii) For the last part of property (ii), we want to show $h(x) \leq \mathbf{s}_{j}^{1}\left(\mathrm{a}_{1}\right)$ whenever $0<j<q+1$ and $x \in S_{2}$. We have $h(x) \leq g(x) \leq w \cdot \mathrm{s}_{q}^{q-1} x$ by definitions (28) and (26), so there are two cases. First, assume $0<j<q$. In this case we note that from $w \in S_{q}$ and (ii) for $S_{q}$ we get $w \leq \mathrm{s}_{j}^{1} \mathrm{a}_{1}$, so $h(x) \leq \mathrm{s}_{j}^{1} \mathrm{a}_{1}$. Suppose $j=q$. In this case we have $x \leq \mathrm{s}_{j}^{1} \mathrm{a}_{1}$ for $0<j<q$ by (ii) for $S_{q}$ since $x \in S_{q}$. In particular, $x \leq \mathrm{s}_{q-1}^{1} \mathrm{a}_{1}$, so $h(x) \leq \mathrm{s}_{q}^{q-1} \mathrm{~s}_{q-1}^{1} \mathrm{a}_{1}=\mathrm{s}_{q}^{1} \mathrm{a}_{1}$. We get the rest of property (ii) by showing $h(x)$ is $(q+1)$-color-ordered and $(q+1)$-covered for every $x \in S_{q}^{w}$. From $x \in S_{q}^{w}$ and property (ii) for $S_{q}$ we know $x$ is $q$-covered, so there are atoms $x_{i, j} \in A t(\mathbf{A})$ such that

$$
x \leq \prod_{0 \leq i<j<q} \mathrm{~s}_{i}^{0} \mathrm{~s}_{j}^{1}\left(x_{i, j}\right) .
$$

Of course, we also know $w \in S_{q}$, so there is a $q$-covering $w_{i, j} \in A t(\mathbf{A}), 0 \leq i<j<$ $q$, of $w$ as well, where

$$
w \leq \prod_{0 \leq i<j<q} \mathbf{s}_{i}^{0} \mathbf{s}_{j}^{1}\left(w_{i, j}\right) .
$$


Let

$$
t_{i, j}= \begin{cases}w_{i, j} & \text { if } 0 \leq i<j<q \\ x_{i, q-1} & \text { if } 0 \leq i<q-1 \text { and } j=q \\ f(x) & \text { if } i=q-1 \text { and } j=q\end{cases}
$$

We shall see that $t_{i, j}$ is a $(q+1)$-covering of $h(x)$. First, note that

$$
\mathbf{s}_{q}^{q-1} x \leq \prod_{0 \leq i<q-1} \mathbf{s}_{i}^{0} \mathbf{s}_{q}^{1}\left(x_{i, q-1}\right)
$$

because if $0 \leq i<q-1$ then $x \leq \mathrm{s}_{i}^{0} \mathrm{~s}_{q-1}^{1}\left(x_{i, q-1}\right)$ by (29), so

$$
\begin{aligned}
\mathrm{s}_{q}^{q-1} x & \leq \mathrm{s}_{q}^{q-1} \mathrm{~s}_{i}^{0} \mathrm{~s}_{q-1}^{1}\left(x_{i, q-1}\right) & & \text { [7, } 1.5 .3] \\
& =\mathrm{s}_{i}^{0} \mathrm{~s}_{q}^{q-1} \mathrm{~s}_{q-1}^{1}\left(x_{i, q-1}\right) & & \text { [7, 1.5.10(iii)] } \\
& =\mathrm{s}_{i}^{0} \mathrm{~s}_{q}^{1}\left(x_{i, q-1}\right) & & \mathrm{c}_{q-1} x_{i, q-1}=x_{i, q-1}, \text { [7, 1.5.11(i)] }
\end{aligned}
$$

Then we have

$$
\begin{aligned}
h(x) & \leq g(x)=w \cdot \mathrm{s}_{q}^{q-1} x \cdot \mathrm{s}_{q-1}^{0} \mathrm{~s}_{q}^{1}(f(x)) \\
& \leq \prod_{0 \leq i<j<q} \mathrm{~s}_{i}^{0} \mathrm{~s}_{j}^{1}\left(w_{i, j}\right) \cdot \prod_{0 \leq i<q-1} \mathrm{~s}_{i}^{0} \mathrm{~s}_{q}^{1}\left(x_{i, q-1}\right) \cdot \mathrm{s}_{q-1}^{0} \mathrm{~s}_{q}^{1}(f(x)) \\
& =\prod_{0 \leq i<j<q} \mathrm{~s}_{i}^{0} \mathrm{~s}_{j}^{1}\left(t_{i, j}\right) \cdot \prod_{0 \leq i<q-1} \mathrm{~s}_{i}^{0} \mathrm{~s}_{q}^{1}\left(t_{i, q}\right) \cdot \mathrm{s}_{q-1}^{0} \mathrm{~s}_{q}^{1}\left(t_{q-1, q}\right) \\
& =\prod_{0 \leq i<j<q+1} \mathrm{~s}_{i}^{0} \mathrm{~s}_{j}^{1}\left(t_{i, j}\right)
\end{aligned}
$$

so $h(x)$ is $(q+1)$-covered.

To show $h(x)$ is $(q+1)$-color-ordered, we assume $0 \leq i<j<k<q+1$ and must show $\mathrm{c}\left(t_{i, j}\right)=\mathrm{c}\left(t_{i, k}\right)$. If $i<j<k<q$ then the first case in (31) applies to both $t_{i, j}$ and $t_{i, k}$, hence $t_{i, j}=w_{i, j}$ and $w_{i, k}=t_{i, k}$, but $\mathrm{c}\left(w_{i, j}\right)=\mathrm{c}\left(w_{i, k}\right)$ because $w$ is color-ordered, so we have $\mathrm{c}\left(t_{i, j}\right)=\mathrm{c}\left(t_{i, k}\right)$. We may therefore assume $k=q$.

We need to observe before going on that if $q>2$, then

$$
\begin{array}{rlrl}
w \leq \mathrm{c}_{q-1} w & =\mathrm{c}_{q-1} x & & \text { property (iii) of } S_{q} \\
& =\mathrm{c}_{q-1}\left(\prod_{0 \leq i<j<q-1} \mathrm{~s}_{i}^{0} \mathrm{~s}_{j}^{1}\left(x_{i, j}\right)\right) & & \text { [7, 1.2.6], (29) } \\
& =\prod_{0 \leq i<j<q-1} \mathrm{~s}_{i}^{0} \mathrm{~s}_{j}^{1}\left(x_{i, j}\right) & \mathrm{c}_{q-1} x_{i, j}=x_{i, j}, q-1 \geq 2
\end{array}
$$

By the uniqueness of coverings this tells us that

$$
w_{i, j}=x_{i, j} \text { if } 0 \leq i<j<q-1 .
$$

If, in addition to $k=q$, we have $i<j<q-1$, then $q>2$ and the first and second cases of (31) apply, so we have $t_{i, j}=w_{i, j}$ and $t_{i, k}=t_{i, q}=x_{i, q-1}$. But $w_{i, j}=x_{i, j}$ by (34). Also, $x$ is color-ordered, so $\mathrm{c}\left(x_{i, j}\right)=\mathrm{c}\left(x_{i, q-1}\right)$, which is equivalent to $\mathrm{c}\left(t_{i, j}\right)=\mathrm{c}\left(t_{i, k}\right)$ by the previous equations.

The final case is that $i<j=q-1$ and $k=q$. The possibilities for $i$ divide into two sub-cases, $i$ is smaller than $q-2$, and $i$ is equal to $q-2$. If $0 \leq i<q-2$ then $i<q-2<q-1$, so $\mathrm{c}\left(t_{i, j}\right)=\mathrm{c}\left(w_{i, q-1}\right)=\mathrm{c}\left(w_{i, q-2}\right)$ since $w$ is color-ordered by 
property (ii) of $S_{q}$, and c $\left(x_{i, q-2}\right)=\mathrm{c}\left(x_{i, q-1}\right)$ since $x$ is color-ordered, but $w_{i, q-2}=$ $x_{i, q-2}$ by (34), so

$$
\begin{aligned}
\mathrm{c}\left(t_{i, j}\right) & =\mathrm{c}\left(w_{i, q-1}\right) & & j=q-1, \\
& =\mathrm{c}\left(w_{i, q-2}\right) & & w \text { is color-ordered } \\
& =\mathrm{c}\left(x_{i, q-2}\right) & & \\
& =\mathrm{c}\left(x_{i, q-1}\right) & & x \text { is color-ordered } \\
& =\mathrm{c}\left(t_{i, k}\right) & & q=k,
\end{aligned}
$$

We are reduced to assuming $i=q-2$, hence

$$
\mathrm{c}\left(t_{i, j}\right)=\mathrm{c}\left(w_{q-2, q-1}\right)=\mathrm{c}\left(x_{q-2, q-1}\right)=\mathrm{c}\left(t_{i, k}\right)
$$

by (34) and the third case in (31).

We have shown that every $h(x)$ constructed from some $x \in S_{q}^{w}$ is non-zero, $(q+1)$ covered, and $(q+1)$-color-ordered. Thus $R$ and all its subsets has property (ii).

To prove property (v) for $R$ (and its subsets), we assume $x, y \in S_{q}^{w}, h(x) \neq h(y)$, $u, v \in A t(\mathbf{A}), h(x) \leq \mathbf{s}_{q}^{1} u, h(y) \leq \mathbf{s}_{q}^{1} v$. We must show $u \neq v$. If we have a $q$-covering of $x$ as in (29), then by (33) we get $u=x_{0, q-1}$ from $h(x) \leq \mathrm{s}_{q}^{1} u$, and, similarly, $v=y_{0, q-1}$ from $h(y) \leq \mathbf{s}_{q}^{1} v$ for some $q$-covering $y_{i, j}$ of $y$. Hence $x \leq \mathbf{s}_{q-1}^{1}\left(x_{0, q-1}\right)$ and $y \leq \mathrm{s}_{q-1}^{1}\left(y_{0, q-1}\right)$, so, by property $(\mathrm{v})$ for $S_{q}^{w}$, we know $x_{0, q-1} \neq y_{0, q-1}$, i.e., $u \neq v$, as desired.

To prove property (vi) for $R$ (and its subsets), we assume $0<j<k<q+1$, $u, v \in A t(\mathbf{A}), x \in S_{q}^{w}$, and $h(x) \leq \mathbf{s}_{j}^{1} u \cdot \mathrm{s}_{k}^{1} v$. If $k<q$, then $u=t_{0, j}=w_{0, j}$ and $v=t_{0, k}=w_{0, k}$ by (33) and (31), but $w \in S_{q}$, so by property (vi) for $S_{q}$, we have $w_{0, j} \neq w_{0, k}$, hence $u \neq v$. Suppose that $k=q$. In this case, by (33) and (31), we again have $u=t_{0, j}=w_{0, j}$ but this time $v=t_{0, q}=x_{0, q-1}$. Hence $w \leq \mathrm{s}_{j}^{1} u$ and $x \leq \mathrm{s}_{q-1}^{1} v$ by (29) and (30). If $j=q-1$ we note that $w \neq x$ since $x \in S_{q}^{w}$, hence $u \neq v$ by property (v) for $S_{q}$, which gives us $t_{0, j} \neq t_{0, q}$, i.e., $u \neq v$. If $j<q-1$ then $v=t_{0, q}=x_{0, q-1} \neq x_{0, j}$ for $0<j<q-1$ by property (vi) for $S_{q}$, applied this time to $x$. But $x_{0, j}=w_{0, j}=t_{0, j}$ by (34) and (31), so again we have $t_{0, q} \neq t_{0, j}$.

We have proved $R$ has properties [(ii), (iii)] (v), and (vi)] and wish to show that $h$ is one-to-one on $S_{q}^{w}$. Assume $x, y \in S_{q}^{w}$ and $x \neq y$. We want to show $h(x) \neq h(y)$. By property (ii) for $S_{q}^{w}, x$ and $y$ have $q$-coverings that include atoms $x_{0, q-1}, y_{0, q-1} \in \operatorname{At}(\mathbf{A})$ satisfying $x \leq \mathrm{s}_{q-1}^{1}\left(x_{0, q-1}\right)$ and $y \leq \mathrm{s}_{q-1}^{1}\left(y_{0, q-1}\right)$. By (31) and (33) these last two equations imply $h(x) \leq \mathrm{s}_{q}^{1}\left(x_{0, q-1}\right)$ and $h(y) \leq \mathrm{s}_{q}^{1}\left(y_{0, q-1}\right)$. From $x \neq y$ we conclude by property (v) for $S_{q}^{w}$ that $x_{0, q-1} \neq y_{0, q-1}$, which implies, by property $(\mathrm{v})$ for $R$, that $h(x) \neq h(y)$, as desired.

Now we want to choose a subset $S_{q+1}$ of $R$ with property (iv) that contains at least $p^{p+1-(q+1)}$ elements. We partition $R$ and let $S_{q+1}$ be the largest piece. Recall from (33) that $h(x) \leq \mathrm{s}_{q-2}^{0} \mathrm{~s}_{q-1}^{1}(f(x))$ for every $x \in S_{q}^{w}$, and $f(x)$ has color $\mathrm{c}(f(x)) \in A t(\mathbf{E})$. For every color $\mathrm{a}_{i}$ we get a piece of $R$, namely

$$
R_{i}:=\left\{h(x): x \in S_{q}^{w}, \mathrm{c}(f(x))=\mathrm{a}_{i}\right\} .
$$

Note that $R$ is the disjoint union of the pieces, the number of pieces is $p$, and $R$ has at least $p^{p+1-q}$ elements because $h$ is one-to-one and $S_{q}^{w}$ has more than $p^{p+1-q}$ elements. Consequently some piece has at least $p^{p+1-q} / p=p^{p-q}$ elements in it, and we let $S_{q+1}$ be any such piece. Thus $S_{q+1}$ has property (i) Every piece has 
property (iv), so in particular $S_{q+1}$ has this property. Finally, as a subset of $R$, $S_{q+1}$ has all the other properties. This completes the construction of the sets $S_{q}$.

Consider what happens when $q=p+1$. We may choose some $x \in S_{p+1}$ because $S_{p+1}$ has at least one element, by property (i). Then $x$ is $(p+1)$-covered, $(p+1)$ color-ordered, and non-zero by property (ii). Let $x$ have $(p+1)$-covering $x_{i, j} \in$ At (A) for $0 \leq i<j<p+1$.

Consider the set $\left\{\mathrm{c}\left(x_{i, p}\right): 0 \leq i<p\right\} \subseteq A t(\mathbf{E})$. Note that $\mathrm{c}\left(x_{0, p}\right)=\mathrm{a}_{1} \neq 1$ ' since $x_{0, p} \leq \mathrm{a}_{1}$ by property (ii). We can also show $\mathrm{c}\left(x_{i, p}\right) \neq 1$ ' for $0<i<p$ because we have, by the covering of $x, x \leq \mathrm{s}_{i}^{1}\left(x_{0, i}\right) \cdot \mathrm{s}_{i}^{0} \mathrm{~s}_{p}^{1}\left(x_{i, p}\right) \cdot \mathrm{s}_{p}^{1}\left(x_{0, p}\right)$ so it follows by [14, Lemma 10] that $\left[x_{0, i}, x_{i, p}, x_{0, p}\right]$ is a cycle, i.e., $x_{0, i} ; x_{i, p} \geq x_{0, p}$. If $\mathrm{c}\left(x_{i, p}\right)=1$ ' then $x_{i, p}=1$ ' and we would get $x_{0, i}=x_{0, p}$, contradicting property [vi) which says $x_{0, i} \neq x_{0, p}$ for $0<i<p$. Thus we know $\mathrm{c}\left(x_{i, p}\right)$ is a diversity atom of $\mathbf{E}$ for $0 \leq i<p$.

The number of diversity atoms in $\mathbf{E}$ is $p-1$, but the size of the index set $\{i: 0 \leq i<p\}$ is $p$. Therefore some atom is repeated, i.e., there are $0 \leq i<j<p$ such that $\mathrm{c}\left(x_{i, p}\right)=\mathrm{c}\left(x_{j, p}\right)$. By the $(p+1)$-color-ordering of $x, \mathrm{c}\left(x_{i, j}\right)=\mathrm{c}\left(x_{i, p}\right)$. Let $u=\mathrm{c}\left(x_{i, j}\right)=\mathrm{c}\left(x_{i, p}\right)=\mathrm{c}\left(x_{j, p}\right)$. We proved above that $u \neq 1$ '. By the covering of $x$ and property (ii) we have $0 \neq x \leq \mathrm{s}_{i}^{0} \mathrm{~s}_{j}^{1}\left(x_{i, j}\right) \cdot \mathrm{s}_{j}^{0} \mathrm{~s}_{p}^{1}\left(x_{j, p}\right) \cdot \mathrm{s}_{i}^{0} \mathrm{~s}_{p}^{1}\left(x_{i, p}\right)$, hence by the definition of $u$ and [14, Lemma 10] we have $0 \neq u ; u \cdot u$. Since $u \neq 1$, this contradicts the assumption that $\mathbf{E}$ has no such diversity atom as the $u$ we have found.

Proof of (ii). Assume to the contrary that $\mathbf{C a}\left(B_{3}(\mathbf{A})\right) \in \mathbf{S N r}_{3} \mathrm{CA}_{p+1}$. Then

$$
\begin{aligned}
\mathbf{A} \cong \mathbf{R a}\left(\mathbf{C a}\left(B_{3}(\mathbf{A})\right)\right) & \in \mathbf{R} \mathbf{a}^{*} \mathbf{S N r}_{3} \mathrm{CA}_{p+1} \\
& =\mathbf{S R a}{ }^{*} \mathrm{Nr}_{3} \mathrm{CA}_{p+1} \\
& =\mathbf{S} \mathbf{R a}^{*} \mathrm{CA}_{p+1}
\end{aligned}
$$

8], defs

contradicting part (i) that says $\mathbf{A} \notin \mathbf{S R a C A}_{p+1}$.

Department of Mathematics, 396 Carver Hall, Iowa State University, Ames, Iowa 50011-2066, USA

E-mail address: maddux@iastate.edu 